\title{
UTILISATION DE L'ENERGIE DES ALIMENTS PAR LES ANIMAUX
}

PAR

ANDRE-M. LEROY

Professeur de Zootechnie à l'I. N. A.

\section{PLAN DU MÉMOIRE}

I. -- Exposé des problèmes concernant la détermination des besoins énergétiques des animaux et la couverture de ces besoins.

II. - Variation de la dépense d'énergie d'un animal avant, pendant et après un repas. Action exercée sur cette dépense par la quantité de matière sèche ingérée.

III. - Exposé des résultats obtenus dans divers pays, par des observateurs différents, au cours d'expériences d'échanges énergétiques, effectuées par les méthodes classiques de calorimétrie directe et indirecte.

IV. - Analyse et comparaison statistiques de ces résultats pour chaque espèce, et, dans le cas d'une même espèce, pour divers expérimentateurs.

V. - Utilisation des données ainsi acquises à la résolution des problèmes d'alimentation et de substitutions alimentaires.

VI. - Conclusion.

Nous savons que la quantité d'énergie métabolisable correspondant à une ration journalière donnée s'obtient en retranchant de l'énergie brute des aliments distribués, telle qu'elle pourrait être obtenue par une expérience de combustion dans une bombe calorimétrique, les quantités d'énergie correspondant respectivement aux excrétions solides, liquides et gazeuses émises en 24 heures, sous la condition formelle que le régime alimentaire étudié n'ait pas subi de modifications pendant la durée de l'observation. Cette quantité d'énergie métabolisable, lorsqu'elle est destinée à un animal domestique donnant des produits utiles à l'homme (viande, lait, œufs, laine) sert à deux fins essentielles - d'une part, elle assure la couverture des besoins énergétiques de l'animal lui-même, c'est-à-dire ceux qu'exigent ses métabolismes, et, d'autre part, elle correspond strictement à la quantité d'énergie accumulée dans les produits obtenus.

Nous savons calculer aisément, à partir de la partie digestible des 
aliments, a quantité d'énergie métabolisable que la somme de ces derniers représente. - D'autre part, connaissant la composition des tissus formés au cours de la croissance et de l'engraissement, ainsi que celles du lait, de l'œuf ou de la laine, il nous est facile de savoir la quantité totale d'énergie correspondant à la production journalière de ces matériaux organiques. L'inconnte $d u$ problème, lorsqu'il s'agit d'alimenter rationnellement un animal, est l'évaluation aussi précise que possible de l'énergie qu'il perd par le jeu de ses divers métabolismes, dans les conditions du milieu où il vit.

Nous savons, grâce à de nombreux expérimentateurs, calculer le besoin énergétique net d'entretien d'un animal, en fonction de son poids vif. La quantité d'énergie d'entretien, exprimée en calories, correspond aux dépenses de chaleur entraînées par le jeu des divers mécanismes vitaux sur un animal en état de jeûne, telles qu'il serait possible de les mesurer au moyen d'un calorimètre.

$\mathrm{Si}$ nous désignons le besoin d'énergie nette d'entretien par $\mathrm{E}$, et la quantité d'énergie calorifique correspondant aux produits qu'il s'agit d'obtenir par P, l'expérience nous apprend qu'une quantité d'énergie métabolisable égale à $\mathrm{E}+\mathrm{P}$ ne suffit pas à placer l'animal étudié dans des conditions d'équilibre énergétique suffisantes. - Avec Rubner, puis KELLNFR, nous avons appris que la consommation des aliments entraîne une augmentation des dépenses métaboliques, d'autant plus grande que la masse des aliments ingérés est elle-même plus considérable. A ce besoin supplémentaire, qui s'ajoute aux besoins du strict entretien, KEILNER a donné le nom d'énergie du travail de la digestion, bien qu'il ne s'agisse pas exclusivement, à proprement parler, d'une perte de calories correspondant au travail mécanique de la mastication et du péristaltisme gastro-intestinal. Si nous désignons par $S$ ce supplément d'énergie, l'équation qui exprime l'équilibre des besoins énergétiques d'un animal donnant des produits peut s'écrire sous la forme suivante, en convenant d'adopter la lettre M pour figurer le besoin d'énergie métabolisable.

$$
\underset{\begin{array}{c}
\text { (Energie utile des } \\
\text { aliments) }
\end{array}}{\mathrm{M}}+\underset{\begin{array}{c}
\text { (Energie dissipée dans } \\
\text { l'atmosphère ambiante) }
\end{array}}{\mathrm{E}+\mathrm{S}} \quad \begin{gathered}
\mathrm{P} \\
\text { (Energie des produits } \\
\text { zootechniques obtenus) }
\end{gathered}
$$

Il est ainsi facile de constater que c'est la quantité $S$ qui demeure la seule inconnue du problème, car nous avons les moyens d'évaluer $\mathrm{M}, \mathrm{E}$, et $\mathrm{P}$, à la condition de connaître la composition et la digestibilité des aliments distribués, le poids vif de 1'animal et la quantité ainsi que la composition des produits qu'il s'agit d'obtenir. Depuis KFILLNER, le besoin combiné d'énergie d'entretien et de production, $\mathrm{E}+\mathrm{P}$, est désigné sous le nom de besoin d'énergie nette. Pour les ruminants, le rapport du besoin d'énergie nette, $\mathrm{E}+\mathrm{P}$, à l'énergie méta- 
bolisable M, varie autour de 57 p. Ioo, selon la composition des mélanges d'aliments distribués. Pour les monogastriques, comme le porc, le lapin et les volailles, ce rapport, beaucoup plus élevé, atteint en moyenne 73 p. Ioo.

Depuis la publication des travaux de KELLNER, 1'habitude a été prise d'exprimer la valeur énergétique des aliments, ainsi que les besoins d'entretien et de production des animaux, en quantités d'énergie nette. L'unité d'énergie nette, adoptée par KELLNER, correspond, comme chacun sait, à la quantité d'énergie capable de satisfaire les besoins nets de production provenant de la consommation par l'animal d'un kilogramme d'amidon. Malheureusement, cette quantité d'énergie nette, exprimée en calories, n'est pas exactement la même, lorsqu'il s'agit d'équilibrer le même besoin, pour les diverses espèces domestiques. Elle n'est pas la même non plus, selon qu'il s'agit de satisfaire le besoin d'entretien, le besoin de croissance, le besoin d'engraissement ou celui de la production laitière. Pour bien comprendre cet état de choses, et en tirer les conséquences qu'il comporte, il est essentiel, à notre avis, d'étudier le mécanisme des pertes d'énergie de l'animal alimenté, dans les conditions normales de son existence.

Le meilleur moyen, pour aborder cette étude, consiste à enregistrer méthodiquement, à des intervalles aussi rapprochés que possible, les quantités d'énergie perdues par l'animal au cours d'une période de 24 heures. Il suffit pour cela d'utiliser la méthode de calorimétrie indirecte dite des échanges gazeux, dont le principe consiste à mesurer pendant un temps donné la consommation d'oxygène et le rejet de gaz carbonique du sujet d'expérience. - Pour éviter l'utilisation du masque respiratoire, qui risque d'apporter du trouble à l'équilibre physiopsychologique de l'animal, et qui ne permet pas surtout de 1'observer pendant qu'il mange, il est préférable, comme nous l'avons fait, d'utiliser la méthode đu confinement, qui consiste à enfermer l'animal dans une enceinte close de capacité réduite, pendant une courte période de temps, et à mesurer la consommation d'oxygène et le rejet de gaz carbonique d'après la variation de composition des gaz de l'enceinte observée au cours de l'expérience. - L'emploi de cette méthode permet de multiplier autant qu'on le désire les prises d'essai, et de connaître ainsi les variations de la dépense énergétique au cours d'une période d'observation qui peut durer au besoin plusieurs jours.

En utilisant cette méthode, nous avons pu montrer que la consommation des aliments s'accompagne immédiatement toujours d'un accroissement considérable de la dépense énergétique. Lorsque deux repas se suivent, la pointe de métabolisme observée au cours du second repas tend toujours à dépasser celle du premier. La figure I ci-jointe, particulièrement démonstrative, représente la variation de la dépense énergétique d'un agneau qui a reçu six repas par jour, entre $9 \mathrm{~h}$ et $2 \mathrm{I} \mathrm{h}$, chacun de ces repas étant composé de $15^{\circ}$ gr de carottes et de 150 gr d'un aliment concentré, apportant au total I50 gr de matière sèche. 
Les mesures de la dépense énergétique ont été au nombre de 15 . Six d'entre elles ont été prises pendant les repas, et cinq ont été prises entre les repas, quelques minutes avant la distribution du repas suivant.

Dans une autre expérience, dont la durée a été d'une semaine, nous avons alimenté une brebis quatre fois par jour, toutes les six heures, en lui faisant absorber chaque fois des repas uniformes, comprenant chacun 420 grammes de matière sèche. I,a figure 2 reproduit graphiquement les résultats moyens des mesures effectuées pendant et entre les repas, rapportés à une période de 24 heures. - Comme les précédents, ces résultats montrent que la pointe de la dépense énergétique, qui accompagne chaque repas, est toujours suivie d'une phase de rémission. Il a été remarquable, au cours de ces essais, de constater que la hausse du métabolisme, qui s'était produite dès le début de chaque repas, s'est maintenue au même niveau maximum pendant toute la durée de la consommation et de la mastication des aliments.

Des phénomènes exactement semblables peuvent être mis en évidence par l'observation d'animaux monogastriques, tels que les porcs et les volailles. La figure 3 montre la variation de la dépense énergétique horaire d'un poulet de I $700 \mathrm{gr}$, recevant trois repas par jour. Nous observons ici, comme précédemment, le net accroissement de métabolisme correspondant à chaque repas, ainsi que la tendance de chacune des deux dernières pointes de métabolisme à dépasser le maximum précédent. - - Nous reproduisons également (fig. 4) les variations de la dépense d'énergie d'un porc alimenté deux fois par jour. Ces observations ont une portée générale. Iilles montrent à l'évidence que la consommation des alinents entraîne, chez tous les animaux, une hausse importante de la consommation d'oxygène. Cette consommation, rapportée à l'unité de temps, est, chez les ruminants, près de trois fois plus forte que la valeur correspondante de la dépense métabolique du strict entretien, mesurée sur l'animal en état de jeûne prolongé. La hausse du métabolisme qui accompagne un repas débute toujours immédiatement, dès que l'animal commence à absorber des aliments et se maintient au même niveau pendant tout le repas, sans subir de diminution sensible. La dépense énergétique qu'elle entraîne qui est en quelque sorte pour l'animal la rançon de la nécessité de se nourrir, se montre d'autant plus considérable que la durée totale des repas pris au cours d'une même journée est plus longue. Pour cette raison, les ruminants, consommateurs ordinaires d'aliments peu digestibles, dont la mastication et la déglutition demandent souvent près de cinq heures, ont un rendement de la transformation de l'énergie métabolisable en énergie nette moins élevé que celui des porcs et des volailles, lesquels peuvent consommer la totalité de leurs aliments quotidiens en une demi-heure à une heure.

Ces observations nous ont conduit à attribuer à la masse de matière 


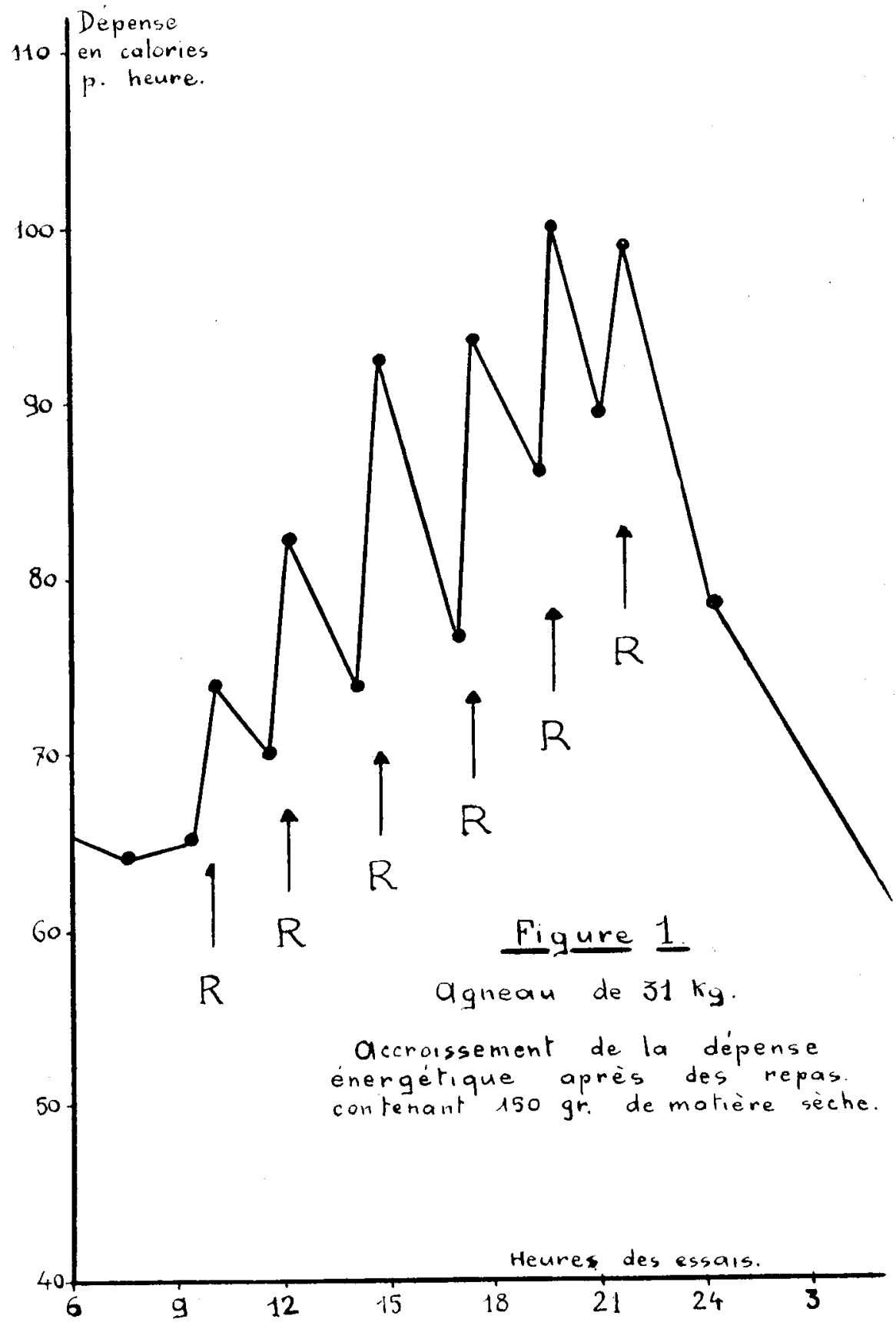




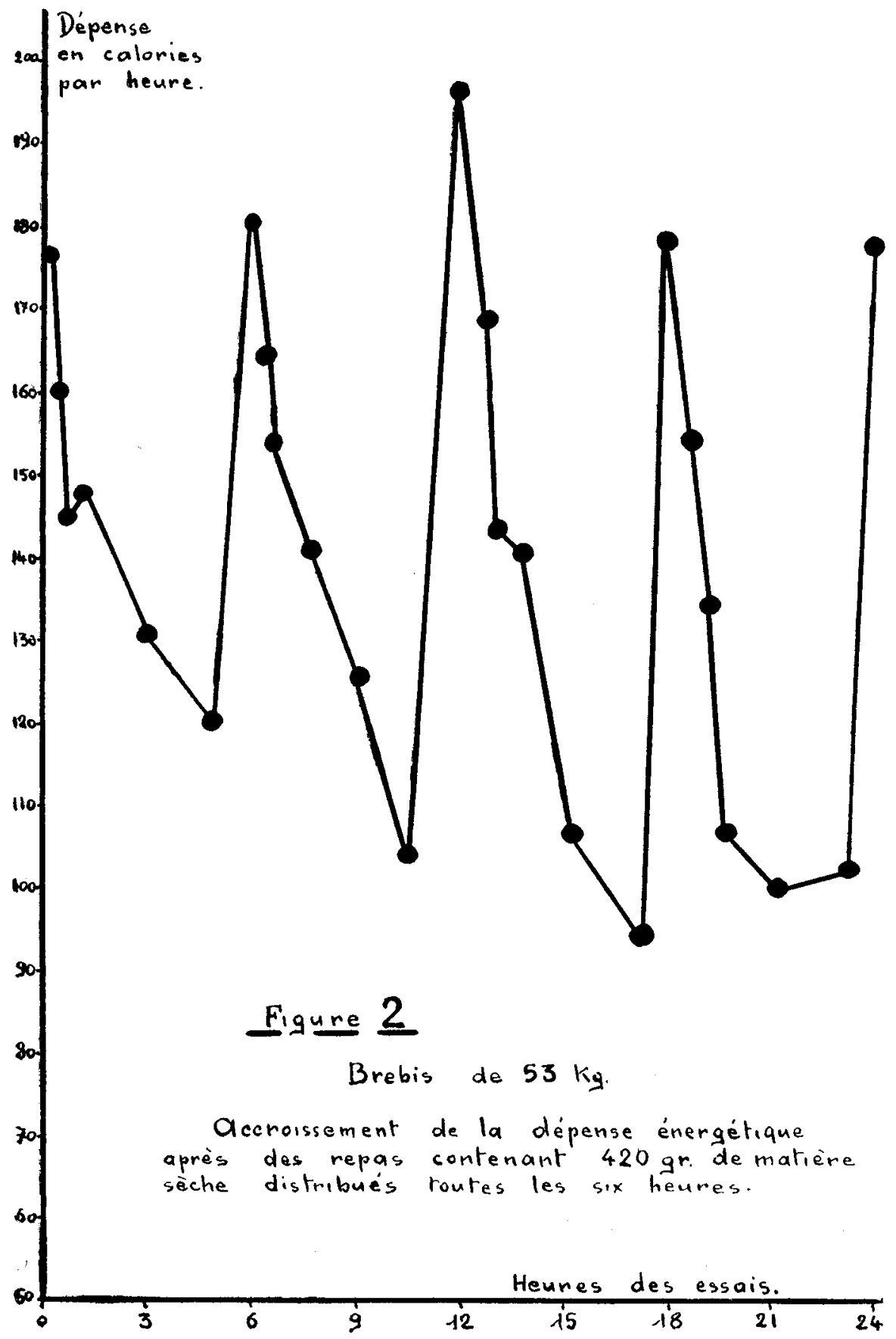




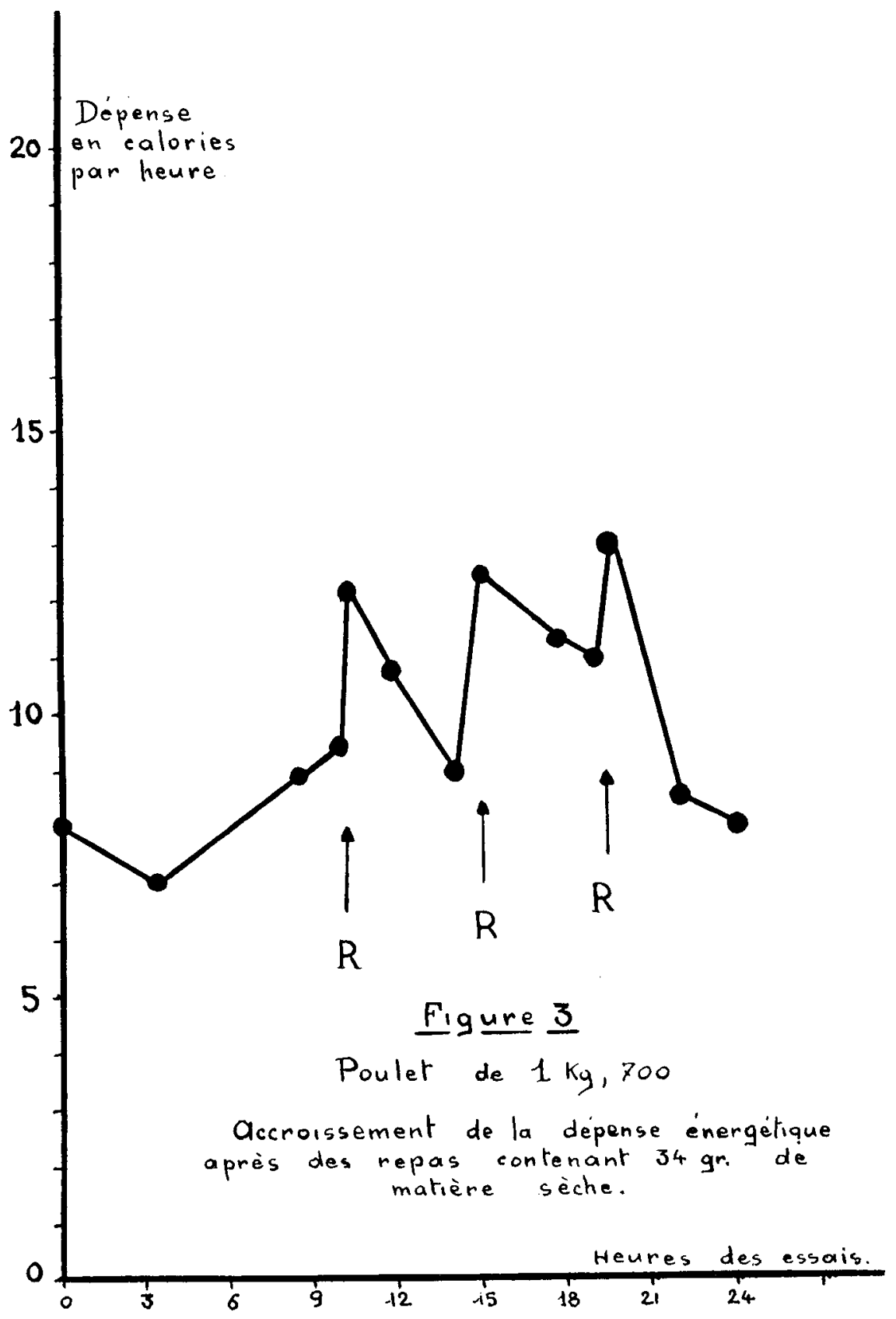




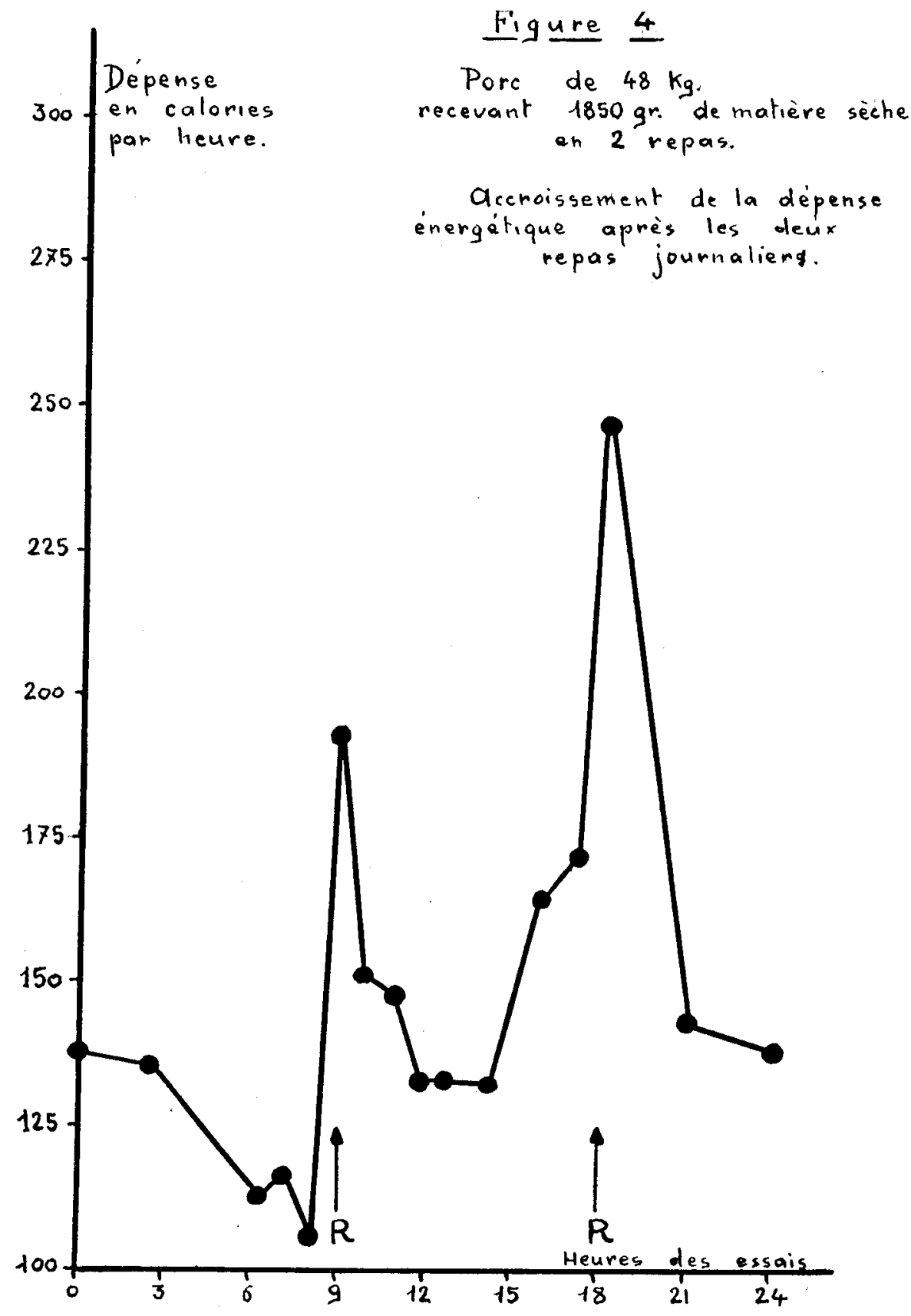


sèche ingérée une importance prépondérante, en ce qui concerne le mode d'utilisation des aliments.

Afin de mettre en évidence cette influence de la matière sèche, nous avons étudié attentivement les résultats expérimentaux publiés depuis le début de ce siècle, ayant pour objet des déterminations précises de balance énergétique effectuées sur des bovins, des ovins, des porcins, des volailles, ainsi que sur des lapins et des rats blancs de laboratoire. Parmi les travaux examinés figurent les mémoires fondamentaux de Kellner et Kohler, de Fingerl,ing, de Forbes et ses collaborateurs, de Mollgaard et de Mittchell et Hamilton. Nous y avons joint nos propres observations effectuées sur des moutons, des porcs et des volailles, ainsi que le remarquable travail d'A. HELL,BERG sur l'alimentation des lapins, et enfin celui de Forbes, Braman et Kriss sur le rat blanc. D'autres résultats rassemblés par nos soins sont venus compléter cette collection de documents. Nous avons donc ainsi réuni 337 séries de données concernant six espèces animales différentes, et se décomposant de la manière ci-après :

\section{'Nableau I}

Nombres d'observahons utilisées, classées par espèce animale

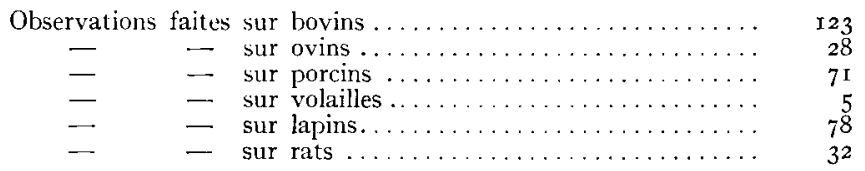

Pour chacune des expériences que nous avons utilisées, nous avons rapproché les données concernant les mesures de la matière sèche ingérée, de l'énergie métabolisable, et de l'énergie de la production, qui correspond, selon le cas, à l'énergie nette de croissance ou d'engraissement, calculée d'après le bilan, ou à la production laitière, ou encore à l'énergie nette combinée des tissus formés et de la quantité du lait produit. La quantité d'énergie métabolisable a été désignée par la lettre $M$, celle de l'énergie nette de la croissance, de l'engraissement et de la production laitière a été désignée par $\mathrm{P}$, tandis que nous avons appelé la quantité de matière sèche consommée, exprimée en grammes, par Ms. Pour déterminer les valeurs de l'énergie nette d'entretien, $\mathrm{F}$, correspondant à chaque essai, nous nous sommes servis du poids moyen de chaque sujet observé au cours de l'expérience et des tables ci-après, reproduites d'après les normes pour l'alimentation énergétique extraites du rapport signé de notre nom figurant dans la publication du Ve Congrès International de Zootechnie (Paris, novembre I949).

Pour la commodité du travail, ces données ont été utilisées par nous sous la forme de graphiques, afin d'éviter des calculs d'interpolation. 


\section{TABLEAU II}

Tables pour la détermination des besoins de l'entretien évalués en grandes calories par 24 heures.

\section{Bovins}

Poids des animaux kg

Ioo............

200. . . . . . .

$300 \ldots \ldots \ldots \ldots$

$400 \ldots \ldots \ldots \ldots$

Poids des animaux

$\mathrm{kg}$

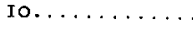

$20 \ldots \ldots \ldots \ldots$

30 .

40 .

Poids des animaux $\mathrm{kg}$

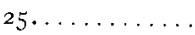

50.

$75 \ldots \ldots \ldots$

$100 \ldots \ldots \ldots . . . .$.

$125 \ldots \ldots \ldots \ldots$

Poids des animaux $\mathrm{kg}$

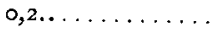

$0,4 \ldots \ldots \ldots \ldots$

$0,6 \ldots$

$\circ, 8 \ldots \ldots \ldots \ldots$

I,O...........

$\mathrm{I}, 2, \ldots \ldots \ldots \ldots$

$1,4 \ldots \ldots \ldots \ldots$

$\mathrm{I}, 6$.

Poids des animalıx $\mathrm{kg}$

I $\ldots \ldots \ldots \ldots$

$\mathrm{I}, 5 \ldots$

$2 \ldots \ldots \ldots \ldots$
Besoins d'en

tretien en cal. Poids des animaux par $24 \mathrm{~h}$.

2080
3500
$475 \circ$
5880

$475^{\circ}$

5880

Ovins

Besoins d'en-

tretien en cal. Poids des animaux par $24 \mathrm{~h}$.

$$
\begin{array}{r}
540 \\
780 \\
960 \\
\text { I } 090
\end{array}
$$

\section{Porcins}

Besoins d'entretien en cal. par $24 \mathrm{~h}$.

$$
\begin{aligned}
& \text { I } 060 \\
& \text { I } 560 \\
& \text { I } 950 \\
& 2240 \\
& 2520
\end{aligned}
$$

\section{Volailles}

Besoins d'entretien en cal. par $24 \mathrm{~h}$.

Poids des animaux $\mathrm{kg}$

27
58
65
80
96
I 10
122
I 36

Besoins d'entretien en cal. par $24 \mathrm{~h}$.

65
89
I 06
I 25

Poids des animaux $\mathrm{kg}$

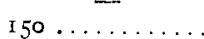

175 .

200 .

225 .

$250 \ldots \ldots . . . .$.

Poid

k

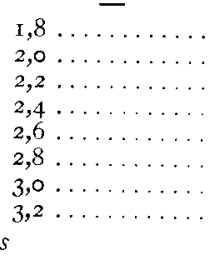

Poids des animaux
$2,5 \ldots \ldots \ldots \ldots$

Rats blancs

Besoins d'entretien en cal. par $24 \mathrm{~h}$.

Poids des animaux

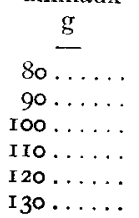

Animaux forte- Animaux ment alimentés rationnés

cal.
I 1,6
I 2,8
I 3,9
I 5, I
I6,3
I 7,5

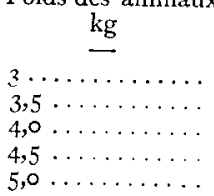

Besoins d'entretien en cal. par $24 \mathrm{~h}$.

$$
\begin{aligned}
& 7000 \\
& 8000 \\
& 8960 \\
& 9900
\end{aligned}
$$

Besoins d'entretien en cal. par $24 \mathrm{~h}$.

$$
\begin{array}{ll}
\text { I } 210 \\
\text { I } 325 \\
\text { I } 435 \\
15 I 5
\end{array}
$$

\begin{tabular}{|c|c|c|}
\hline $\begin{array}{l}\text { Poids des } \\
\text { animaux } \\
\mathrm{g}\end{array}$ & $\begin{array}{c}\text { Animaux forte- } \\
\text { ment alimentés } \\
\text { cal. }\end{array}$ & $\begin{array}{l}\text { Animaux } \\
\text { rationnés } \\
\text { cal. }\end{array}$ \\
\hline $140 \ldots \ldots$ & I 8,7 & $\bar{I} 7,2$ \\
\hline${ }^{1} 5^{\circ} \ldots \ldots$ & 19,9 & 18,4 \\
\hline I $60 \ldots$. & $21, \mathrm{Y}$ & 19,5 \\
\hline I $70 \ldots \ldots$ & 22,2 & 20,8 \\
\hline $180 \ldots \ldots$ & 23,5 & 22,0 \\
\hline $190 \ldots .$. & 24,6 & 23,0 \\
\hline $200 \ldots \ldots$ & 25,8 & 24,2 \\
\hline
\end{tabular}

Besoins d'entretien en cal. par $24 \mathrm{~h}$.

$$
\begin{aligned}
& 2760 \\
& 3000 \\
& 3260 \\
& 3460 \\
& 3630
\end{aligned}
$$

Besoins d'entretien en cal. par $24 \mathrm{~h}$.

$$
\begin{aligned}
& 148 \\
& 162 \\
& 176 \\
& 182 \\
& 198 \\
& 203 \\
& 216 \\
& 228
\end{aligned}
$$

Besoins d'entretien en cal. par $24 \mathrm{~h}$.

$$
\begin{aligned}
& -141 \\
& \text { I } 60 \\
& \text { I } 78 \\
& \text { I } 95 \\
& 211
\end{aligned}
$$

Besoins d'entretien en cal. par $24 \mathrm{~h}$. 
Seul, le tableau des normes d'entretien correspondant aux rats blancs de laboratoire n'a pas été reproduit dans la publication du Congrès de Paris de r949. Les données de ce tableau ont été extraites d'un important travail de Brody, publié en juin I930, et de celui de Forbes, Kriss et MILlER, analysé en détail par nos soins. Flles montrent avec évidence que la mesure du métabolisme basal du rat, effectuée de $\mathrm{I} 7$ à 24 heures après la fin du dernier repas, donne des résultats légèrement différents, selon que l'on effectue la mesure sur des rats ayant reçu auparavant une ration de strict entretien, ou qu'on l'entreprend après avoir laissé pendant plusieurs jours les animaux consommer à volonté leur nourriture. Dans le deuxième cas, les résultats obtenus, pour des animaux de poids identiques, dépassent d'environ ro p. Ioo ceux du premier, comme l'indique notre tableau de normes.

Afin d'éviter l'influence de causes étrangères à l'action que nous voulions étudier, nous avons pris le soin de vérifier que les animaux dont nous examinions le métabolisme pourraient se maintenir en équilibre sans être mis dans1'obligation de faire appel à leurs réserves de matières azotées et de matières grasses, ce qui nous a conduit à éliminer toutes les expériences au cours desquelles les animaux présentaient un bilan énergétique fortement négatif.

De même, pour éviter les effets d'une alimentation azotée surabondante, nous avons écarté les essais pendant lesquels les animaux en période de croissance ou d'engraissement recevaient des quantités de matières protéiques digestibles sensiblement supérieures à $\mathrm{I} g$ par kilogramme de poids vif. Ainsi, par exemple, nous n'avons pas tenu compte des expériences de KELLNER et KOHLER au cours desquelles les bœufs utilisés par ces auteurs avaient reçu de fortes quantités de gluten. Nous avons également omis volontairement, pour une raison analogue, quelques-uns des résultats obtenus par FINGERLING. On sait, en effet, que les animaux qui ingèrent une quantité de matières azotées dépassant celle qui est indiquée par les normes usuelles éliminent par l'urine l'azote excédentaire, ce qui entraîne un accroissement de la dépense d'énergie calorifique, dont 1'ordre de grandeur est de 8,4 calories par gramme d'azote ainsi éliminé, et contribue à diminuer le rapport normal de l'énergie nette à l'énergie métabolisable, comme l'ont signalé de nombreux auteurs, et tout particulièrement MoligaArd. Quelques-uns des résultats de Keliner, provenant d'essais au cours desquels il avait été mélangé aux rations distribuées des quantités fort élevées de matière grasse, n'ont pas été non plus retenues par nous, parce que nous avons supposé que les conditions d'ingestion d'un régime alimentaire si éloigné de l'alimentation habituelle pouvaient influencer défavorablement les phénomènes que nous cherchions à mettre en évidence.

Pour chacun des essais retenus, le lecteur trouvera, avec les références bibliographiques, les résultats de la différence $\mathrm{M}-(\mathrm{E}+\mathrm{P})$ entre l'énergie métabolisable et l'énergie nette, calculée par gramme de matière sèche ingérée. 
Première série

\section{Expériences sur bovins en période de croissance et d'engraissement}

\begin{tabular}{|c|c|c|c|c|c|c|}
\hline No de l'essai & $\begin{array}{c}\text { Énergie } \\
\text { métabolisable } \\
\text { M } \\
\text { cal. }\end{array}$ & $\begin{array}{c}\text { Mat. sèche } \\
\text { consommée } \\
\text { Ms } \\
\text { g }\end{array}$ & $\begin{array}{c}\text { Energie de } \\
\text { l'entretien } \\
\text { J } \\
\text { cal. }\end{array}$ & $\begin{array}{c}\text { Énergie de } \\
\text { la production } \\
\text { P } \\
\text { cal. }\end{array}$ & $\begin{array}{c}\mathrm{M}-(\mathrm{E}+\mathrm{P}) \\
\text { cal. }\end{array}$ & $\frac{M-(\mathrm{E}+\mathrm{P})}{\mathrm{Ms}}$ \\
\hline
\end{tabular}

I. - Expériences de KeLLner et KOHLER

(Landw. Versuchs Staiionen, 53, I ; 1900)

\begin{tabular}{|c|c|c|}
\hline Bœuf & A...... & I6 368 \\
\hline - & & 工 6389 \\
\hline$\ldots$ & C I & 26789 \\
\hline- & C II .... & 29928 \\
\hline 一 & C $\Pi \ldots$ & $33 \circ 85$ \\
\hline - & & $15 \times 35$ \\
\hline - & & 15918 \\
\hline - & 20. & 17440 \\
\hline & 3. & 19666 \\
\hline - & 4 II & $1879 \mathrm{I}$ \\
\hline & 5 II $a$ & 2048 \\
\hline- & $5 \mathrm{II} b$ & 20706 \\
\hline & $611 b$. & 21152 \\
\hline - & 6 III . . & $2435^{\circ}$ \\
\hline & D I. & $2595^{8}$ \\
\hline & $\mathrm{DI}$ & 30594 \\
\hline & E 1 & 26397 \\
\hline & E & 25327 \\
\hline -... & F II & $\begin{array}{l}23400 \\
23417\end{array}$ \\
\hline$\ldots$ & F IlI . & 1768 \\
\hline
\end{tabular}

\begin{tabular}{r|r|r}
6 I 70 & 8190 & I 549 \\
7120 & 8310 & 832 \\
10960 & 8040 & 7478 \\
I2 539 & 8510 & 8886 \\
I 2597 & 8650 & 9748 \\
6600 & 8020 & 1487 \\
6540 & 8430 & 1684 \\
7210 & 8690 & 957 \\
7810 & 8470 & 3007 \\
7770 & 8300 & 1761 \\
7940 & 8190 & 4268 \\
7910 & 8380 & 4014 \\
7970 & 8720 & 4062 \\
8900 & 8880 & 5414 \\
10707 & 8810 & 6617 \\
12383 & 9230 & 8769 \\
10694 & 8710 & 5918 \\
I0 737 & 9150 & 4336 \\
10142 & 7830 & 4593 \\
10539 & 8155 & 4230 \\
7076 & 7960 & 2061
\end{tabular}

\begin{tabular}{|c|c|}
\hline 6629 & 1,07 \\
\hline 7247 & 1,02 \\
\hline If $27 \mathrm{I}$ & 1,03 \\
\hline I $253^{2}$ & 1,00 \\
\hline$I_{4} 687$ & 1,16 \\
\hline 5628 & 0,85 \\
\hline 5804 & 0,89 \\
\hline 7793 & $I, 08$ \\
\hline $8 \mathbf{I} 39$ & $I, 04$ \\
\hline 8730 & I, I3 \\
\hline 8030 & $\mathrm{I}, \mathrm{OI}$ \\
\hline $8^{812}$ & 1,05 \\
\hline $937^{\circ}$ & $\mathrm{I}, \mathrm{I} 7$ \\
\hline $1005^{6}$ & I,I 3 \\
\hline $1053 \mathrm{I}$ & 0,98 \\
\hline I2 595 & 1,02 \\
\hline II 769 & I, IO \\
\hline I I $84 \mathrm{I}$ & I, IO \\
\hline 10142 & $\mathrm{~J}, 09$ \\
\hline I I O3 & $\mathrm{I}, 04$ \\
\hline 7659 & 1,07 \\
\hline
\end{tabular}

Bæuf F IV ... 22993

$\begin{array}{r}\text { FVI ... } \\ \text { - GI . } 22563 \\ \hline\end{array}$

- G11..... 23258

- G III ... 17376

- GIV ... 23030

- H I.... 2 I 973

- H II ... 25446

-- H III ... 24042

- H IV ... I 7443

- II V .... 26409

- HVI... 23 I02

- H VII.. 26133

- J I .... $2263 \mathrm{I}$

- J III ... $2262 \mathrm{I}$

- JIV.... I7 87 I

-- J V..... 26433

Landw. Versuchs Stationen, 53, I900).

\begin{tabular}{|c|c|c|c|c|}
\hline 8830 & 8 I 80 & 5388 & 9425 & 1,07 \\
\hline 8893 & $855^{\circ}$ & 4628 & 9385 & 105 \\
\hline 10 495 & 8165 & $368+$ & I0 865 & $I, 03$ \\
\hline IO IIO & 8300 & 3938 & II 020 & $\mathrm{I}, 08$ \\
\hline 7141 & 8210 & I 778 & $73^{88}$ & $r, 03$ \\
\hline 8880 & 8430 & $53^{67}$ & 9233 & $\mathrm{I}, \mathrm{O}_{4}$ \\
\hline I0 283 & 8440 & 2737 & Io $79^{8}$ & I,05 \\
\hline I0 204 & $853^{\circ}$ & $625^{8}$ & 10 $65^{8}$ & $\mathrm{~J}, 04$ \\
\hline 8834 & $86 \mathbf{I} 5$ & 5808 & 9619 & 1,09 \\
\hline $685^{8}$ & 8660 & 2057 & 6726 & 0,98 \\
\hline 9640 & 8960 & 7873 & $957^{6}$ & $\mathrm{I}, \infty \mathrm{O}$ \\
\hline 8731 & 9080 & 6379 & 7643 & 0,8 \\
\hline Io 434 & 9320 & 5673 & I I I 40 & 1,06 \\
\hline $1024 \mathrm{I}$ & $8 I 45$ & 3565 & 10921 & I,06 \\
\hline 8455 & 8260 & 4996 & 9365 & I, I I I \\
\hline 6907 & 8335 & 2302 & 7234 & $\mathrm{I}, 04$ \\
\hline $965^{8}$ & 8605 & 7140 & I0 688 & $\mathrm{I}, \mathrm{IO}$ \\
\hline 8712 & 8760 & $456 \mathrm{I}$ & 9614 & $\mathrm{I}, \mathrm{IC}$ \\
\hline
\end{tabular}

2. - Expériences de FingerLing et coll.

(Landw. Versuchs Stationen, II3, I-68; I932).
Bœuf ro I.... I9 I 2 I
7780
8700
1702
8719
I, IO

(Landw. Versuchs Stationen, 114, 1-II2 ; 1933).

\begin{tabular}{|c|c|c|c|c|c|c|c|}
\hline Bœuf & U I. & 2. 506 & 8700 & $845^{\circ}$ & 4924 & 8132 & 0,94 \\
\hline- & U II . & 27862 & Io 853 & 8710 & 8882 & I0 $27^{\circ}$ & 0,94 \\
\hline 一 & U III & 28203 & Io 835 & 9000 & 8319 & I0 884 & I,, 00 \\
\hline- & U IV & $215^{8 \mathrm{I}}$ & $875^{x}$ & 8990 & $45^{2 \mathrm{I}}$ & 8340 & 0,95 \\
\hline 一 & V I $\ldots$ & $22 \times 52$ & 8777 & 8000 & 5909 & 8243 & 0,94 \\
\hline - & V III ... & 29184 & Io 823 & 8590 & 9528 & 1 1066 & 1,02 \\
\hline 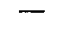 & V IV & 21819 & $87 \times 2$ & 8610 & 4806 & 8403 & 0,97 \\
\hline
\end{tabular}




\begin{tabular}{|c|c|c|c|c|c|c|}
\hline No de l'essai & $\mid \begin{array}{c}\text { Énergie } \\
\text { métabolisable } \\
\text { (M) } \\
\text { cal. }\end{array}$ & $\begin{array}{l}\text { Mat. seche } \\
\text { consommét } \\
\text { (Ms) } \\
\text { g }\end{array}$ & $\begin{array}{l}\text { Énergie de } \\
\text { l'entretien } \\
\text { (Ii) } \\
\text { cal. }\end{array}$ & $\left\{\begin{array}{c}\text { Énergie de la } \\
\text { production } \\
\text { (P) } \\
\text { cal. }\end{array}\right.$ & $\mid \begin{array}{c}\mathrm{M}-(\mathrm{E}+\mathrm{P}) \\
\mathrm{cal} .\end{array}$ & $\frac{\mathrm{M}-(\mathrm{E}+\mathrm{P})}{\mathrm{Ms}}$ \\
\hline
\end{tabular}

(Lanilw. Versuchs Stationen, I 6 , I-63; r933).

\begin{tabular}{|c|c|c|c|c|c|c|c|}
\hline Bouf & U IV & $2 I 58 I$ & $875 \mathrm{I}$ & 8960 & $425 \mathrm{I}$ & 8370 & 0,96 \\
\hline- & U VI & 28720 & $1094 \mathrm{I}$ & 9 2 го & $788 \mathrm{r}$ & II 629 & $\mathrm{I}, 06$ \\
\hline - & U VII & 21847 & 8865 & 9540 & 3633 & 8674 & 0,98 \\
\hline 一 & V IV & 21819 & 8712 & 8610 & 4806 & 8403 & 0,06 \\
\hline - & V VI & 28749 & I0 887 & 9000 & 8263 & II 486 & $I, 05$ \\
\hline - & V VII & 21760 & 8787 & 9180 & 4007 & 8573 & 0,98 \\
\hline
\end{tabular}

(Land\%o. Versuchs. Stationen, I 20-12 I, I-I4I ; 1934)

\begin{tabular}{|c|c|c|c|c|c|c|c|}
\hline Bouf & I7 I & 21425 & 8257 & $835^{\circ}$ & 5130 & 7945 & 0,96 \\
\hline - & $17 \mathrm{~V}$ & $2849 \mathrm{I}$ & Io 885 & 9000 & 7203 & I 2288 & $\mathrm{I}, \mathrm{I} 3$ \\
\hline - & I7 VIHI & 21607 & 8339 & 9400 & 3660 & 8547 & 1,02 \\
\hline - & W I . & 21902 & 8255 & 8410 & +285 & 9307 & $\mathrm{I}, \mathrm{I} 2$ \\
\hline - & W III & 27719 & I0 265 & 9000 & 6084 & I 2635 & I, I 2 \\
\hline - & W IV & 21903 & $8 \cdot 289$ & 9010 & $35^{85}$ & 9308 & I, 12 \\
\hline - & X I & 21534 & 8216 & $83^{60}$ & 4567 & 8607 & $\mathrm{I}, 04$ \\
\hline - & X IV & 2 I 939 & 8285 & 9020 & $396 \mathrm{I}$ & 8958 & 1,08 \\
\hline
\end{tabular}

3. - Expériences de Forbes E. B., Braman W. W. et Kriss M.

(Journal of Agr. Res., 35, 233, 1928).

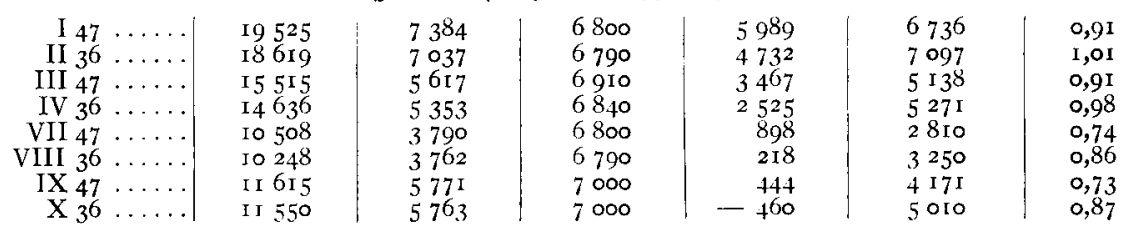

(Journal of Agr. Res., 40, 37 ; 1930).

\begin{tabular}{|c|c|c|c|c|c|c|}
\hline I 60 & 7545 & 2828 & 4840 & 403 & 2302 & 0,82 \\
\hline II 57 & 8243 & 3085 & 5460 & 399 & 2384 & 0,78 \\
\hline III $60 \ldots \ldots$ & I I 24 I & 4237 & $5 \mathrm{I} 20$ & $260 \mathrm{I}$ & 3520 & 0,83 \\
\hline IV 57 & 12469 & 4612 & 5700 & 3176 & 3593 & 0,78 \\
\hline$V 60 \ldots$ & 14902 & 5704 & 5400 & 3925 & 5577 & 0,98 \\
\hline VI 57 & 16363 & 6233 & 5900 & 4484 & 5979 & 0,96 \\
\hline VII 60 & 19467 & $75^{20}$ & 5810 & $55^{83}$ & 8074 & 1,07 \\
\hline VIII 57 & 20855 & 8057 & $635^{\circ}$ & 6882 & 7623 & 0,95 \\
\hline IX 60 & 23896 & 9489 & 6 IIO & $77^{6} 4$ & 10022 & 1,06 \\
\hline XII 57 (1). & 10188 & 5155 & 6120 & $50 \mathrm{I}$ & 3567 & $0,7 \mathrm{I}$ \\
\hline XIII 60 (I) & 9658 & 5013 & 6000 & I67 & 49 I & 0,7 \\
\hline
\end{tabular}

(Journal of Agr. Res., 43, II, IOI5: I93I).

\begin{tabular}{|c|c|c|c|c|c|c|}
\hline$I_{1} z_{7} \ldots$ & $9 I 14$ & 4827 & 5500 & $-\quad 42$ & 3656 & 0,76 \\
\hline III 17 & 7255 & $2 \mathrm{I} 74$ & 5190 & -205 & 2270 & $\mathrm{I}, 04$ \\
\hline $\mathrm{V} 17$ & 7706 & 2994 & 5100 & 525 & $208 \mathrm{I}$ & 0,70 \\
\hline IV 85 & 8790 & $443^{\mathrm{I}}$ & 5120 & -602 & 4272 & 0,96 \\
\hline
\end{tabular}

(Journal of Agric. Res., 46, 8, 753 ; г933).

\begin{tabular}{|c|c|c|c|c|c|c|}
\hline III A & 8767 & 3954 & 5800 & -409 & 3376 & 0,85 \\
\hline IV B $\ldots$ & 7646 & 3610 & $5 \circ 3 \circ$ & -639 & 3255 & 0,90 \\
\hline$V A \ldots \ldots$ & I2 659 & $5 \mathrm{I} 03$ & 6000 & $25^{2} \mathrm{I}$ & $4 \mathbf{I} 38$ & $0,8 I$ \\
\hline VI B . . & II I 55 & 4661 & $5 \pm 40$ & I 937 & $4 \circ 78$ & 0,87 \\
\hline VII A & 8980 & $397^{\circ}$ & $595^{\circ}$ & 212 & 2818 & $0,7 \mathrm{I}$ \\
\hline VIII & 7410 & 3428 & 5050 & -275 & 2635 & 0,77 \\
\hline
\end{tabular}




\begin{tabular}{|c|c|c|c|c|c|c|}
\hline No de l'essai & \begin{tabular}{|c|} 
Énergie \\
métabolisable \\
M \\
cal.
\end{tabular} & $\begin{array}{c}\text { Mat. sèche } \\
\text { consommée } \\
\text { Ms } \\
\mathrm{g}\end{array}$ & $\begin{array}{c}\text { Énergie de } \\
\text { l'entretien } \\
\text { E } \\
\text { cal. }\end{array}$ & $\begin{array}{l}\text { Énergie de } \\
\text { la production } \\
\mathrm{P} \\
\text { cal. }\end{array}$ & $\begin{array}{c}\mathrm{M}-(\mathbf{E}+\mathrm{P}) \\
\text { cal. }\end{array}$ & $\frac{\mathrm{M}-(\mathrm{E}+\mathrm{P})}{\mathrm{Ms}}$ \\
\hline
\end{tabular}

\begin{tabular}{|c|c|c|c|c|c|c|}
\hline IX A $\ldots$ & I $289 \mathrm{I}$ & 5206 & $6 \times 20$ & 2222 & 4549 & 0,87 \\
\hline$X$ B & Io 656 & 4530 & 5250 & 1253 & 4 I 53 & 0,92 \\
\hline XI A & 10978 & $480 I$ & 6210 & 607 & $4 \mathrm{I} 6 \mathrm{I}$ & 0,87 \\
\hline $\begin{array}{r}\text { XIIB } \ldots \\
\text { XIIIB } \ldots .\end{array}$ & $\begin{array}{r}9025 \\
1255^{8}\end{array}$ & $\begin{array}{l}4 \mathrm{I}_{48} \\
53^{1} 5\end{array}$ & $\begin{array}{l}5330 \\
5600\end{array}$ & $\begin{array}{r}350 \\
1760\end{array}$ & $\begin{array}{l}4045 \\
5108\end{array}$ & 0,97 \\
\hline
\end{tabular}

4. - Expériences de H. H. Mitchell et T. S. Hamilton

(Journal of Agr. Res., 45, 3 ; 1932).

\begin{tabular}{|c|c|c|c|c|c|c|}
\hline $\begin{array}{l}\text { Pleine ration... } \\
4 / 5 \text { de la pl. rat. } \\
3 / 5 \text { de la pl. rat. } \\
2 / 5 \text { de la pl. rat. }\end{array}$ & $\begin{array}{l}2988 \mathrm{I} \\
24320 \\
18982 \\
13563\end{array}$ & $\begin{array}{l}\text { II } 229 \\
8975 \\
6806 \\
4644\end{array}$ & $\begin{array}{ll}7 & 880 \\
8 & 130 \\
8 & 210 \\
8 & 100\end{array}$ & $\begin{array}{l}10969 \\
8398 \\
4562 \\
1044\end{array}$ & $\begin{array}{l}11032 \\
7792 \\
6210\end{array}$ & $\begin{array}{l}0,98 \\
0,87 \\
0,91\end{array}$ \\
\hline & & & & I 044 & 4419 & 0,95 \\
\hline
\end{tabular}

5. - Expériences de Meigs et Grant.

(Proc. Amer. Soc. Animal Prod., I8; 1936).

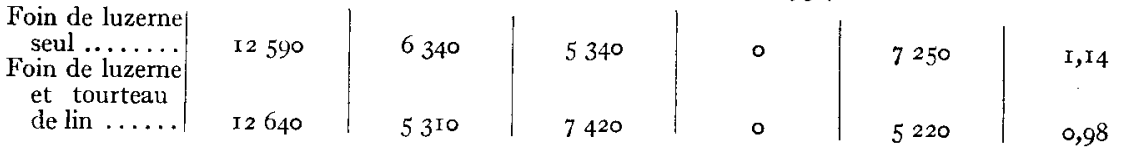

6. - Expériences de K. L. Baxter sur veaux de lait.

(Journal of Nutrition, 6,$12 ; 1952)$.

\begin{tabular}{|c|c|c|c|c|c|c|}
\hline $\begin{array}{l}\mathbf{I} \ldots \ldots \ldots \ldots \\
2 \ldots \ldots \ldots \ldots\end{array}$ & $\begin{array}{l}2801 \\
5969\end{array}$ & $\begin{array}{l}53^{2} \\
959\end{array}$ & $\begin{array}{l}\text { I } 608 \text { (I) } \\
\text { I } 980 \text { (I) }\end{array}$ & $\begin{array}{r}73^{\circ} \\
33^{1}\end{array}$ & $\begin{array}{l}46_{3} \\
95^{8}\end{array}$ & $\begin{array}{l}0,87 \\
\mathrm{I}, 00\end{array}$ \\
\hline
\end{tabular}

7. - Expériences de A. M. Leroy et Z. Zeiter sur veau de lait.

(Annales de Zootechnie, I, 6r-77; 1952).

\begin{tabular}{|c|c|c|c|c|c|c|}
\hline 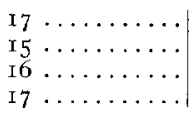 & $\begin{array}{l}477 \mathrm{I} \\
3420 \\
4882 \\
673 \mathrm{I}\end{array}$ & $\begin{array}{r}938 \\
680 \\
\text { I } 200 \\
\text { I } 251\end{array}$ & $\begin{array}{l}\text { I } 520(2) \\
\text { I } 530 \\
\text { I } 710 \\
\text { I } 770\end{array}$ & 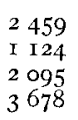 & $\begin{array}{r}792 \\
766 \\
\text { I } 017 \\
\text { I } 283\end{array}$ & $\begin{array}{l}0,85 \\
\text { I, I 2 } \\
0,85 \\
1,02\end{array}$ \\
\hline
\end{tabular}

8. - Expériences de MollgaARd sur vaches laitières.

(Futternugslehre des Milchviehs, Ed. Schaper, Hannover, r929).

\begin{tabular}{|c|c|c|c|c|c|c|}
\hline & & & & & & \\
\hline $\begin{array}{l}40 \mathrm{I} \ldots \ldots \ldots \\
40 \mathrm{II} \ldots \ldots\end{array}$ & $\begin{array}{l}23562 \\
23867\end{array}$ & $\begin{array}{l}8867 \\
8860\end{array}$ & $\begin{array}{l}6000 \\
6000\end{array}$ & Io 518 & 7044 & 0,80 \\
\hline $\begin{array}{llll}40 & 11 & \ldots & \ldots \\
46 & \ldots & \ldots & \ldots\end{array}$ & $\begin{array}{l}23007 \\
24974\end{array}$ & $\begin{array}{r}0000 \\
10153\end{array}$ & $\begin{array}{l}0000 \\
56 \mathrm{IO}\end{array}$ & 8508 & 9359 & $I, 05$ \\
\hline $48 \ldots \ldots \ldots$ & 43215 & I5 5I & 7150 & $\begin{array}{l}\text { 10 } 950 \\
2 \text { I } 574\end{array}$ & $\begin{array}{r}84 \mathrm{I} 4 \\
\mathrm{I} 52 \mathrm{I}\end{array}$ & 0,83 \\
\hline $49 \ldots \ldots \ldots$ & 29693 & II 646 & 5820 & I 2736 & $\begin{array}{ll}15 & 021 \\
11 & 137\end{array}$ & $\begin{array}{l}1,02 \\
0,96\end{array}$ \\
\hline $8 I \ldots$ & $3^{\circ} 33^{2}$ & I I 975 & $765^{\circ}$ & I I 59 & II 523 & 0,97 \\
\hline $55 \cdots \cdots \cdots \cdots$ & $2667 \mathrm{I}$ & 9917 & 7020 & 10622 & 9029 & 0,91 \\
\hline $78 \cdots \cdots \cdots$ & $3 \circ 833$ & 12085 & 7610 & $\times 2087$ & II I 36 & 0,92 \\
\hline $50 \ldots$ & 30007 & I I 639 & 59 Io & I3 I 46 & I0 $95 \mathrm{I}$ & 0,94 \\
\hline $\begin{array}{r}2 \\
53\end{array}$ & 21923 & 9388 & 5890 & 7867 & 8166 & 0,87 \\
\hline $63 \ldots \ldots \ldots$ & $\begin{array}{l}25970 \\
24064\end{array}$ & $\begin{array}{l}9717 \\
9645\end{array}$ & $\begin{array}{l}7020 \\
5600\end{array}$ & 9582 & 9374 & 0,97 \\
\hline $5 I \ldots \ldots$ & 31659 & II 544 & 6420 & $\begin{array}{r}1321 \\
14740\end{array}$ & $\begin{array}{r}9137 \\
10490\end{array}$ & 0,95 \\
\hline $63 \ldots$ & $2440 I$ & 9690 & 5600 & Io 772 & 8029 & $\begin{array}{l}0,91 \\
0,83\end{array}$ \\
\hline 65 engr. & 23546 & 9440 & 5910 & 8289 & 9347 & 0,99 \\
\hline
\end{tabular}

(I) L'Énergie de l'entretien a été dircetement mesurée.

(2) L'Énergie de l'entretien a été directement mesurée. 


\section{Deuxième série}

\section{ESPËE OVINE - CROISSANCE - ENGRAISSEMENT - PRODUCTION LAITIERE}

\begin{tabular}{|c|c|c|c|c|c|c|}
\hline No de l'essai & $\begin{array}{c}\text { Énergie } \\
\text { métabolisable } \\
\mathrm{M} \\
\text { cal. }\end{array}$ & $\begin{array}{c}\text { Mat, sèche } \\
\text { consommée } \\
\text { Ms } \\
\text { g }\end{array}$ & $\begin{array}{c}\text { Énergie de } \\
\text { l'entretien } \\
\text { E } \\
\text { cal. }\end{array}$ & $\begin{array}{l}\text { Energie de } \\
\text { la production } \\
\text { P } \\
\text { cal. }\end{array}$ & $\mid \begin{array}{c}M-(E+P) \\
\text { cal. }\end{array}$ & $\frac{\mathrm{M}-(\mathrm{E}+\mathrm{P})}{\mathrm{Ms}}$ \\
\hline
\end{tabular}

9. - Expériences de Leroy et ZeLter (données en instance de publication). I. - Agneaux alimentés exclusivement avec le lait matemel.

\begin{tabular}{|c|c|c|c|c|c|c|}
\hline I $\ldots \ldots \ldots$ & 1031 & I $7 \mathrm{I}$ & 498 & $35 \mathrm{I}$ & 182 & I,07 \\
\hline $2 \ldots \ldots \ldots$ & $93^{6}$ & I 52 & $52 \mathrm{I}$ & 257 & $15^{8}$ & $I, 04$ \\
\hline $3 \ldots \ldots$ & I 680 & 250 & 565 & 846 & 269 & 1,07 \\
\hline $4 \ldots$ & 880 & 143 & 448 & 262 & 170 & I, I 9 \\
\hline $5 \cdots$ & 796 & I35 & 490 & 162 & I 44 & $\mathrm{I}, 07$ \\
\hline $6 \ldots \ldots$ & $85^{\circ}$ & I 36 & 474 & $23^{2}$ & I 44 & $\mathrm{I}, 06$ \\
\hline $7 \ldots$ & I 037 & 167 & $3^{68}$ & 504 & 165 & 0,99 \\
\hline $8 \ldots \ldots$ & 904 & I 46 & 392 & 354 & I58 & $\mathrm{I}, 08$ \\
\hline $9 \ldots$ & 909 & 146 & 423 & 335 & I $5 \mathrm{I}$ & $I, 03$ \\
\hline $10 \ldots \ldots$ & 734 & 124 & 400 & 164 & 170 & 1,37 \\
\hline II $\ldots \ldots \ldots \ldots$ & $93^{8}$ & r 53 & 424 & $3^{82}$ & $13^{2}$ & 0,8 \\
\hline 13 & I 286 & 232 & 540 & 528 & 218 & 0,94 \\
\hline
\end{tabular}

II. - Agneaux en cours de sevrage et agneaux sevrés.

\begin{tabular}{|c|c|c|c|c|c|c|}
\hline \multicolumn{7}{|l|}{ Avant sevr.: } \\
\hline $23 \ldots \ldots \ldots$ & I 933 & 577 & 746 & $55^{8}$ & 629 & $I, 09$ \\
\hline $25 \ldots \ldots \ldots \ldots$ & 2384 & 525 & 766 & 1163 & 455 & 0,89 \\
\hline $26 \ldots \ldots \ldots$ & 1845 & 546 & 840 & 516 & 489 & 0,90 \\
\hline $27 \ldots \ldots \ldots \ldots$ & I 555 & 407 & 888 & 246 & 421 & $\mathrm{r}, 03$ \\
\hline $28 \ldots \ldots \ldots$ & $25^{18}$ & $83^{6}$ & 948 & 622 & $94^{8}$ & $\mathrm{I}, \mathrm{I} 3$ \\
\hline Apr. sevr. : & & & & & & \\
\hline $29 \ldots \ldots \ldots$ & I 864 & 728 & 997 & 22 & 845 & $\mathrm{I}, \mathrm{I} 6$ \\
\hline$\ldots \ldots$ & 2002 & 696 & I 003 & $49^{6}$ & $5 \circ 3$ & 0,72 \\
\hline $3 \pi$. & 2079 & 817 & I 034 & 491 & 854 & $\mathrm{I}, \mathrm{04}$ \\
\hline
\end{tabular}

III. - Brebis à l'engraisstment.

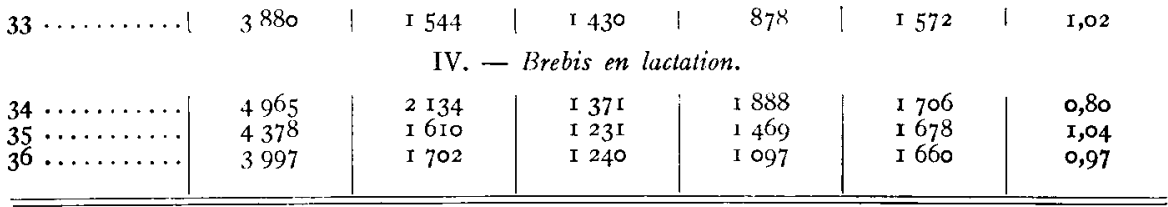

Io. - Expériences de G. Chariet-Léry, A.-M. Leroy et S. Z. Zelter, sur la croissance d'agneaux Ile-de-France.

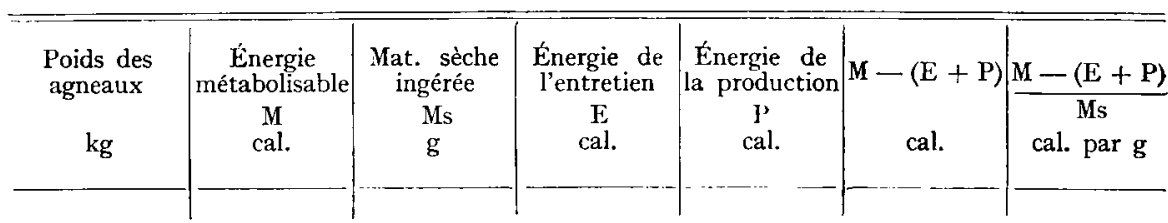

(Annales de Zootechnie, 3, I69-187; 1954).

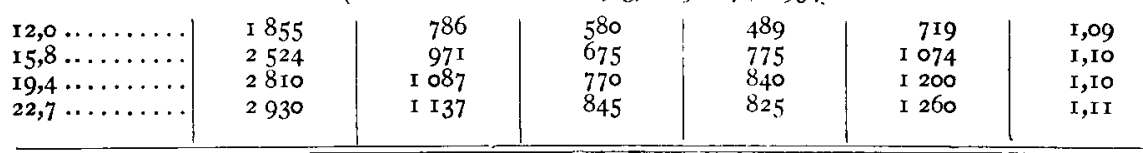


Troisième série

EXPERIENCES SUR PORCS

\begin{tabular}{|c|c|c|c|c|c|c|}
\hline No de l'essai & \begin{tabular}{|c|} 
Énergie \\
métabolisable \\
M \\
cal.
\end{tabular} & $\begin{array}{l}\text { Mat. sèche } \\
\text { ingéréc } \\
\text { Ms } \\
g\end{array}$ & $\begin{array}{c}\text { Energit de } \\
\text { lentreticn } \\
\text { E } \\
\text { cal. }\end{array}$ & $\begin{array}{l}\text { Finergie de } \\
\text { la production } \\
\text { I } \\
\text { cal. }\end{array}$ & $\mid \begin{array}{c}\mathrm{M}-(\mathrm{l}-\mathrm{P}) \\
\text { (al. }\end{array}$ & $\frac{\mathrm{M}-(\mathrm{E}+\mathrm{P})}{\mathrm{Ms}}$ \\
\hline
\end{tabular}

I . .... Expériences de Fingerling.

(Landia. T'ersuchs Stationen, I I.3, $1:$ I932).

Porc 3 :

Per. $4 \cdots \cdots \cdots \mid \quad 5042$

Per. $6 \ldots \ldots \ldots \mid 6399$

Per. $7 \ldots \ldots$ 4... 4976

Porc I :

Per. $2 \ldots$.

Per. $3 \ldots \ldots$

Porc 3 :

Per. 4 ...

Porc XVI :

Per. I ......

Per. $2 \ldots \ldots \ldots$

Per. $3 \ldots \ldots$.

Per. $5 \ldots . .$. .

Porc XVII :

Per. $I \ldots \ldots$.

Per. $2 \ldots \ldots \ldots$

Per. $3 \ldots \ldots$.

Per. 5

$$
\begin{aligned}
& 5086 \\
& 6718 \\
& \\
& 5042 \\
& 6662 \\
& \\
& 4297 \\
& 4557 \\
& 3275 \\
& 4232 \\
& 4357
\end{aligned}
$$

4297

4557

3275

$423^{2}$

$455^{\circ}$

3215

$43^{67}$
Per. $5 \ldots \ldots \ldots$

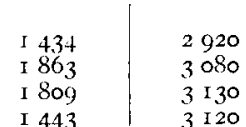

3120

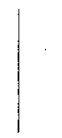

954
2143
1632
948

I 168
I 439
I 637
908

$0,8 \mathrm{I}$

0,77

0,90
0,63

\begin{tabular}{|c|c|c|c|c|c|}
\hline $\begin{array}{l}5086 \\
6718\end{array}$ & $\begin{array}{l}\text { I } 434 \\
\text { I } 863\end{array}$ & $\begin{array}{l}3060 \\
3130\end{array}$ & $\begin{array}{r}796 \\
\text { I } 985\end{array}$ & $\begin{array}{l}\text { I } 230 \\
1603\end{array}$ & $\begin{array}{l}0,86 \\
0,86\end{array}$ \\
\hline $\begin{array}{l}5042 \\
6662\end{array}$ & $\begin{array}{l}\text { I } 4,34 \\
\text { I } 863\end{array}$ & $\begin{array}{l}2920 \\
3080\end{array}$ & $\begin{array}{r}954 \\
2143\end{array}$ & $\begin{array}{l}\text { I } 168 \\
\text { I } 4.39\end{array}$ & $\begin{array}{l}0,81 \\
0,77\end{array}$ \\
\hline
\end{tabular}

(Landw. Versuchs Stationen, 113, 273; 1932).

(Lantr". Versuchs Staitonen, 114 ; I933).

Porc 6 :

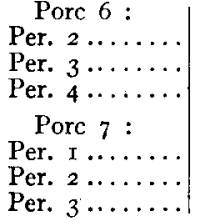

i

\begin{tabular}{r|r|r} 
I 316 & I 780 & I 357 \\
I 306 & I 880 & I 63 I \\
960 & 1975 & $45^{2}$ \\
I 314 & 2170 & 859 \\
& & \\
I 316 & I 660 & I 652 \\
I 306 & I 780 & I 78 I \\
960 & I 875 & 640 \\
I 3I & 2080 & I 175
\end{tabular}

(Landa. Verstuchs Stationen, I 16, I).

4821
4185
4636
4701
5366
4745

1

$\begin{array}{ll}\text { I } & 410 \\ \text { I } & 219 \\ 1 & 391 \\ \text { I } & 387 \\ \text { I } & 586 \\ \text { I } & 394\end{array}$

\begin{tabular}{ll|l}
2 & I 30 & \\
2 & 200 & I 852 \\
2 & 260 & I 202 \\
I 523 \\
2 & 130 & \\
2 & I 779 \\
2 & 240 & 2 \\
2 & 330 & I 552
\end{tabular}

(Landre. Versuchs Stationen, I18-119, 307).

Per. I...... 5093

Per. $2 \ldots \ldots \ldots . \quad 5742$

Per. $3 \ldots \ldots \ldots \mid 569$

\begin{tabular}{l|l|l} 
I 424 & 2 I $5 \circ$ & I 86 \\
I 605 & $225 \circ$ & 2 I 6 \\
1601 & 2280 & 205
\end{tabular}

(Landiv, Versuchs Stationen, 120-12 г, 1 ; 1934).

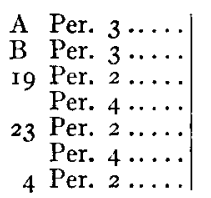

5194
5155
5052
4817
5022
4818
6085

$\begin{array}{ll}\text { I } & 482 \\ 1 & 482 \\ \text { I } & 497 \\ \text { I } & 445 \\ 1 & 497 \\ 1 & 445\end{array}$

2400
2410
1880
$215 \circ$
1870
2140

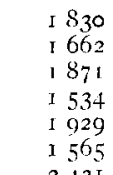

I $76 \mathrm{I}$

2520

\begin{abstract}
2431
\end{abstract}

1160
I 046
848
I 203
1045
989
700
I 112

0,88

0,80

0,88

0,91

0,80

0,76

0,73

12. - Expériences de LERoY et coll. (données non publićes), I940.

Porc $A:$
Per. $2 \ldots \ldots$

Per. $3 \ldots \ldots \ldots$

Per. $4 \ldots \ldots \ldots$

5889
9628

6989

I 2 OI 7

Per. $6 \ldots . .$.

1 2765

$\begin{array}{ll}\text { I } & 380 \\ 1 & 600 \\ 1 & 975 \\ 2 & 160 \\ 2 & 220\end{array}$

$\begin{array}{ll}2 & 219 \\ 5 & 038 \\ 2 & 223 \\ 5 & 547 \\ 7 & 644\end{array}$

I, 06

0,96

0,87

0,97 


\begin{tabular}{|c|c|c|c|c|c|c|}
\hline No de l'essai & $\begin{array}{c}\text { Énergie } \\
\text { métabolisable } \\
\text { M } \\
\text { cal. }\end{array}$ & $\begin{array}{c}\text { Mat. sèche } \\
\text { ingérée : } \\
\text { Ms }_{5} \\
\text { g }\end{array}$ & $\begin{array}{c}\text { Énergie de } \\
\text { l'entretien } \\
\text { E } \\
\text { cal. }\end{array}$ & $\begin{array}{c}\text { Énergie de } \\
\text { la production } \\
\mathrm{P} \\
\text { cal. }\end{array}$ & $\begin{array}{c}\mathrm{M}-(\mathrm{E}+\mathrm{P}) \\
\mathrm{cal} .\end{array}$ & $\frac{\mathrm{M}-(\mathrm{E}+\mathrm{P})}{\mathrm{Ms}}$ \\
\hline
\end{tabular}

I 2. - Expériences de Leror et coll. (domées non publiées) i940 (suite)

Porc B :

\begin{tabular}{|c|c|c|c|c|c|c|}
\hline $\begin{array}{c}\text { Pore } B \text { : } \\
\text { Per. } 2 \ldots \ldots \ldots\end{array}$ & 5149 & 2015 & I $3^{80}$ & 1 826 & I 943 & 0,96 \\
\hline Per. $4 \ldots \ldots$. & 7487 & 2623 & 2080 & 2620 & 2787 & 1,06 \\
\hline $\begin{array}{r}\text { Porc }(\mathrm{:} \\
\text { Per. } 2 \ldots \ldots\end{array}$ & $6 \pm 55$ & 2023 & I 300 & 2840 & 2015 & 1,00 \\
\hline I'er. $3 \ldots$ & 7 I 65 & $244^{2}$ & 1520 & 3653 & I 992 & 0,82 \\
\hline Per. $4 \ldots \ldots$ & 9375 & $33^{82}$ & I 790 & $532 I$ & 2264 & 0,67 \\
\hline Per. $5 \ldots$. & II 005 & 3934 & 2020 & 5348 & 3637 & 0,92 \\
\hline Per. 6. & I 2 OI 4 & 4,424 & $2 \mathbf{I} 35$ & 6518 & $33^{6 r}$ & $0,7^{6}$ \\
\hline Per. $7 .$. & 12997 & +466 & 2190 & 7015 & 3792 & 0,85 \\
\hline
\end{tabular}

13. - Expériences de Leroy et Léry.

(Annales Agronomiques, $\mathrm{I}^{\mathrm{er}}$ trimestre 1946).

Porc de :

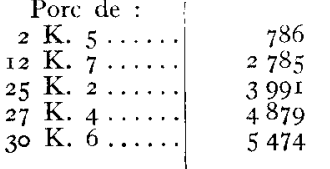

I 63
815
I $55^{\circ}$
I 610
I 800

$\begin{array}{rr}208 \\ 645 \\ \text { I } & 060 \\ \text { I } & \text { I } 20 \\ \text { I } & 200\end{array}$

437
1335
1578
2163
2509

I 41
805
I 353
I. 596
I 765

0,86

0,99

0,87

0,99

0,98

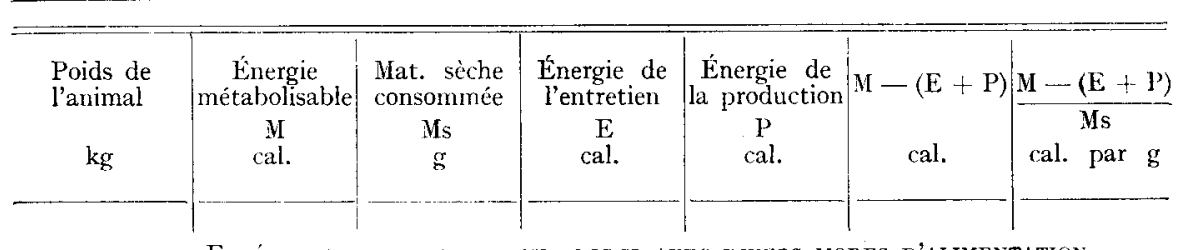

14. - EXPÉRIENCES EXTENSIVES SUR PORCS AVEC DIVERS MODES D'ALIMENTATION

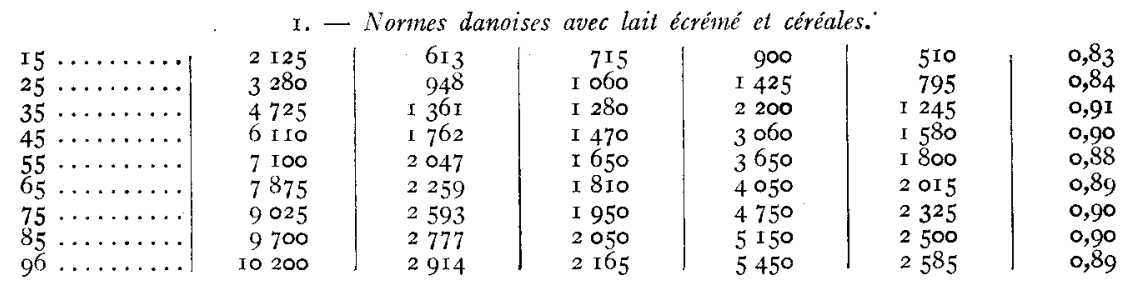

2. - Normes françaises avec sérum de fromagerie.

(Guittonneau et Leroy. Le Porc, par A.-M. Leroy, librairie Hachette).

\begin{tabular}{|c|c|c|c|c|c|c|}
\hline $40 \ldots \ldots \ldots$ & $568_{4}$ & I. 710 & I 400 & 2625 & I 659 & 0,97 \\
\hline$\ldots \ldots$ & 7366 & 2220 & I $73^{\circ}$ & 3509 & $2 \mathrm{I} 27$ & 0,96 \\
\hline $80 \ldots$ & 8770 & 2640 & 2020 & $4 \times 47$ & 2603 & 0,98 \\
\hline $100 \ldots$ & Io 483 & 3240 & 2240 & 5240 & 3003 & 0.93 \\
\hline
\end{tabular}

3. - Expériences avec avoine.

(Leroy et ZeLTER. - Rapports d'expériences sur l'Alimentalion du bétail;

Conseil supérieur de l'Élevage, Paris, 1936).

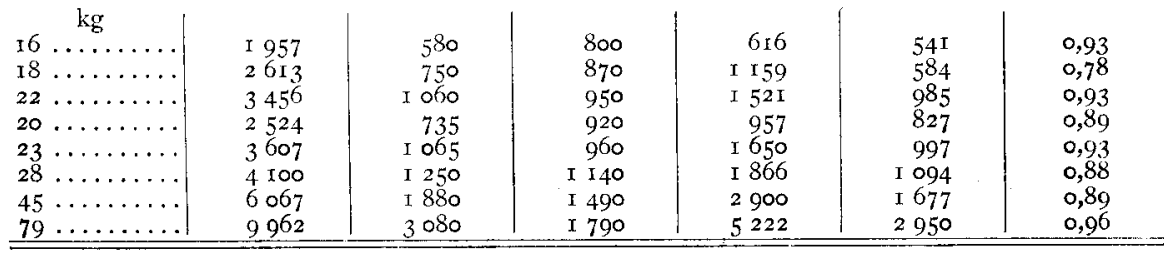




\section{Quatrième série}

\section{ESSAIS SUR LAPINS}

I7. - Expériences suédoises d'A. HellberG.

(A. Hellberg - Metabolism of rabbits at different planes of nutrition, ALMQUist et WikselLs, Uppsala I 949).

\begin{tabular}{|c|c|c|c|c|c|c|c|}
\hline No de l'essai & $\begin{array}{l}\text { Poids du } \\
\text { sujet } \\
\quad \mathrm{kg}\end{array}$ & $\begin{array}{c}\text { Énergie } \\
\text { métabo- } \\
\text { lisable } \\
\text { M } \\
\text { cal. }\end{array}$ & $\begin{array}{c}\text { Mat. sèche } \\
\text { ingérée }\end{array}$ & $\begin{array}{l}\text { Énergie de } \\
\text { l'entretien } \\
\text { E } \\
\text { cal. }\end{array}$ & $\mid \begin{array}{c}\text { Énergie de } \\
\text { la produc } \\
\text { tion } \\
\\
\mathrm{P} \\
\text { cal. }\end{array}$ & $\begin{array}{c}a \\
+ \\
a \\
1 \\
\pm \\
\text { cal. }\end{array}$ & 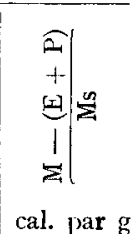 \\
\hline
\end{tabular}

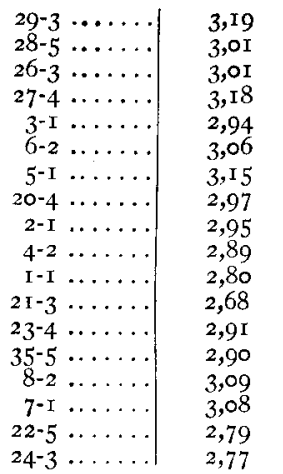

372
348
344
331
308
301
300
261
275
247
267
229
243
214
205
206
179
183

Première partie :

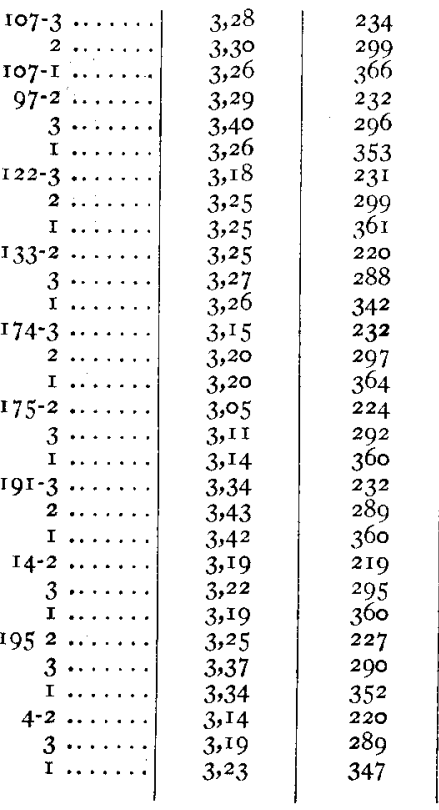

\begin{tabular}{|c|c|}
\hline I 29 & \\
\hline 123 & \\
\hline 120 & \\
\hline I I 5 & \\
\hline 107 & \\
\hline 107 & \\
\hline 103 & \\
\hline 90 & \\
\hline 89 & \\
\hline 89 & \\
\hline 88 & \\
\hline $8 \mathrm{I}$ & \\
\hline 80 & \\
\hline 74 & \\
\hline $7 \mathrm{I}$ & \\
\hline 71 & \\
\hline $\begin{array}{l}63 \\
62\end{array}$ & \\
\hline
\end{tabular}

\begin{tabular}{l|r} 
I48 & I25 \\
I40 & II3 \\
I40 & 108 \\
I48 & 93 \\
I39 & 77 \\
I43 & 76 \\
I46 & 63 \\
I39 & 58 \\
I39 & 63 \\
I38 & 29 \\
I36 & 63 \\
I28 & 33 \\
I38 & 41 \\
I38 & 25 \\
I44 & 0 \\
I43 & 5 \\
I32 & -8 \\
I32 & 10
\end{tabular}

\begin{tabular}{l|l}
99 & 0,77 \\
95 & 0,77 \\
96 & 0,80 \\
90 & 0,78 \\
88 & 0,82 \\
82 & 0,77 \\
$9 \mathrm{I}$ & 0,88 \\
64 & 0,71 \\
73 & 0,82 \\
80 & 0,90 \\
68 & 0,77 \\
68 & 0,84 \\
64 & 0,80 \\
$5 \mathrm{I}$ & 0,69 \\
$6 \mathrm{I}$ & 0,86 \\
58 & 0,82 \\
47 & 0,75 \\
$4 \mathrm{I}$ & 0,66
\end{tabular}

Deuxième partie :

\begin{tabular}{|c|c|c|c|c|}
\hline 75 & I $5 \mathrm{I}$ & 6 & 77 & 1,02 \\
\hline 98 & I 52 & 46 & 101 & 1,03 \\
\hline I 20 & I 50 & 86 & 130 & 1,08 \\
\hline 75 & 152 & 14 & 66 & 0,88 \\
\hline 98 & I 56 & 56 & 84 & 0,86 \\
\hline 120 & 150 & 90 & $\mathrm{II} 3$ & 0,94 \\
\hline 75 & I 47 & -8 & 84 & 1,12 \\
\hline 98 & 150 & $3^{8}$ & I I I & $\mathrm{I}, \mathrm{I} 3$ \\
\hline 120 & 150 & 82 & 129 & 1,08 \\
\hline 75 & 150 & 8 & 62 & 0,82 \\
\hline $9^{8}$ & I $5 \mathrm{I}$ & 52 & 85 & 0,87 \\
\hline 120 & I 50 & 78 & I I 4 & 0,95 \\
\hline 75 & I45 & 9 & 78 & $\mathrm{I}, 04$ \\
\hline $9^{8}$ & $14^{8}$ & $5^{6}$ & 93 & 0,95 \\
\hline 120 & I 48 & 98 & I 18 & 0,98 \\
\hline 75 & I 43 & 14 & 67 & 0,89 \\
\hline 98 & $\times 45$ & 53 & 94 & 0,96 \\
\hline 120 & I 45 & 98 & I I 7 & 0,97 \\
\hline 75 & I 54 & 4 & 74 & 0,99 \\
\hline 98 & 157 & 53 & 79 & $0,8 \mathbf{I}$ \\
\hline I 20 & I 57 & 83 & I 20 & $1, \infty$ \\
\hline 75 & I 47 & 5 & 67 & 0,89 \\
\hline 98 & I 48 & 66 & 81 & 0,83 \\
\hline I 20 & I 47 & 102 & I I I & 0,92 \\
\hline 75 & I $5^{\circ}$ & 12 & 65 & 0,87 \\
\hline 98 & I5 55 & 55 & 80 & 0,82 \\
\hline I 20 & 154 & 79 & 1 I9 & 0,99 \\
\hline 75 & I 45 & I 2 & 63 & 0,84 \\
\hline 98 & I 47 & 57 & 85 & 0,87 \\
\hline 120 & 148 & 79 & I 20 & $I, \infty$ \\
\hline
\end{tabular}




\begin{tabular}{|c|c|c|c|c|c|c|c|}
\hline No de l'essail & $\begin{array}{l}\text { Poids du } \\
\text { sujet } \\
\\
\text { kg }\end{array}$ & $\begin{array}{c}\text { Énergie } \\
\text { métabo } \\
\text { lisable } \\
\text { M } \\
\text { cal. }\end{array}$ & $\begin{array}{c}\text { Mat. sèche } \\
\text { ingérée }\end{array}$ & \begin{tabular}{|} 
Énergie de \\
l'entretien \\
E \\
cal.
\end{tabular} & $\begin{array}{c}\text { Énergie de } \\
\text { la produc } \\
\text { tion } \\
\\
\mathbf{P} \\
\text { cal. }\end{array}$ & $\begin{array}{l}\text { ar } \\
+ \\
\text { xy } \\
1 \\
z \\
\text { cal. }\end{array}$ & $\begin{array}{l}\begin{array}{c}a \\
+ \\
E \\
1 \\
z\end{array} \mid \\
\text { cal. par g }\end{array}$ \\
\hline
\end{tabular}

\begin{tabular}{|c|c|c|}
\hline $185-3 \ldots \ldots$ & 3,30 & 224 \\
\hline $2 \ldots \ldots$ & $3,3^{8}$ & 292 \\
\hline $1 \ldots \ldots$ & 3,47 & $3^{62}$ \\
\hline $184-2 \ldots \ldots$ & 3,24 & 2 I 2 \\
\hline $3 \ldots \ldots$ & 3,32 & 282 \\
\hline I $\ldots \ldots$ & 3,43 & 35 I \\
\hline $12-3 \ldots \ldots$ & 3, I 5 & 225 \\
\hline $2 \ldots \ldots$ & $3, \mathrm{I} 8$ & 292 \\
\hline I $\ldots \ldots$ & 3,10 & $35^{I}$ \\
\hline
\end{tabular}

Deuxiême partie (suite) :

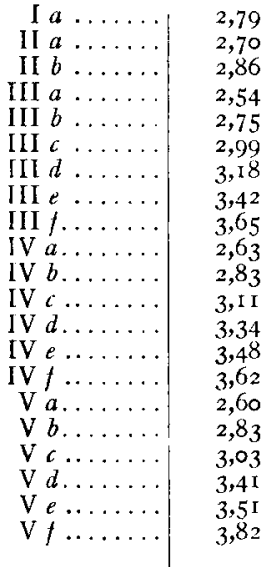

\begin{tabular}{l|l|l}
305 & 104 & 132 \\
305 & 103 & 129 \\
300 & 106 & 135 \\
295 & 109 & 123 \\
295 & 104 & 131 \\
293 & 101 & 140 \\
292 & 108 & 148 \\
284 & 104 & 157 \\
294 & 104 & 165 \\
316 & 103 & 126 \\
318 & 99 & 134 \\
318 & 106 & 145 \\
312 & 103 & 154 \\
311 & 103 & 158 \\
309 & 103 & 164 \\
308 & 103 & 125 \\
317 & 103 & 133 \\
303 & 103 & 142 \\
312 & 103 & 157 \\
315 & 103 & 160 \\
307 & 103 & 172 \\
& &
\end{tabular}

$\circ$
$5^{6}$
$9^{8}$
6
$5^{6}$
93
5
$5^{1}$
90

72
80
106
56
74
I 11
75
94
I 14

0,96

\begin{tabular}{r|}
75 \\
98 \\
I 20 \\
75 \\
98 \\
$\mathbf{1} 20$ \\
75 \\
98 \\
I 20
\end{tabular}

\begin{tabular}{l|} 
I 52 \\
I 56 \\
I 58 \\
I 50 \\
I 52 \\
I 57 \\
I 45 \\
I 47 \\
I 47
\end{tabular}

0,88

0,75

0,76

0,84

I,, 00

0,96

Troisième partie :

Au cours de l'impression de ce travail, nous avons eu connaissance d'une thèse publiée à Zurich, jar J. Barboriak, sur le métabolisme énergétique du lapin. Au moyen des données de cet auteur, obtenues à la suite de $\mathrm{I}_{4}$ expériences effectuées avec 5 sujets, nourris d'aliments progressivement enrichis en cellulose brute, nous avons pu calculer un rapport $\frac{M-(E+P)}{M s}$ de $0,90 \pm 0,053 . \mathbf{L a}$ différence de cette moyenne avec celle de l'ensemble des résultats provenant des expériences de Hellberg, qui est de $0,040 \pm 0,054$, est dépourvue de signification.

Nous nous permettons de regretter que le travail de $J$. BARBORIAK nous soit parvenu trop tard pour nous permettre de faire figurer les données que nous avons pu en extraire à la suite des précédentes. 
Cinquième série:

ESSAIS SUR VOLAILLES

DÉTERMINATION EXPÉRIMENTALE DE L'TANERGIE NETTE DISPONIBLE POUR LES VOLAILLES PROVENANT DE L'INGESTION DE DIVERSES CÉRÉALES

I8. - DÉTERMINATION DE LA VALEUR ÉNERGÉTIQUE NETTE DE L'ORGE ET DU MaIs. (G. S. Fraps. Texas Agr. Exp. Station Bull. 625 ; 1942).

\begin{tabular}{|c|c|c|c|c|c|}
\hline Désiqu & $\begin{array}{l}\text { Énergie métabo- } \\
\text { lisable p. } 100 \mathrm{~g} \\
\text { de produit }\end{array}$ & Mat. siche & $\begin{array}{l}\text { Énergie nette } \\
\text { correspondante } \\
\text { mesurée }\end{array}$ & & M \\
\hline du p & $\begin{array}{l}\text { ingéré } \\
\text { M } \\
\text { cal. }\end{array}$ & $\begin{array}{c}\text { correspondante } \\
\text { Ms } \\
\mathrm{g}\end{array}$ & $\begin{array}{l}\text { expérimentalement } \\
\text { Ne } \\
\text { cal. }\end{array}$ & $\begin{array}{l}\text { Différence } \\
M-\mathrm{Ne} \\
\text { cal. }\end{array}$ & cal. par g \\
\hline arge -... & ${ }_{282,5}$ & $\frac{-}{87,2}$ & $\overline{197}$ & $\overline{85,5}$ & $\frac{-}{0,98}$ \\
\hline Mais. & 338 & 88,2 & 243 & 95 & ז,07 \\
\hline
\end{tabular}

I9. - DéterMination DE LA VALEUR ÉNERGÉtioue NeTte DE L'AVOINE (F. Bachmann. - C. R. du VIII e Congrès international d'Aviculture).

\begin{tabular}{|c|c|c|c|c|c|}
\hline & $\begin{array}{c}\text { Énergie métabo- } \\
\text { lisable p. Ioo g } \\
\text { de produit }\end{array}$ & Nen & $\begin{array}{l}\text { Énergie nette } \\
\text { correspondante } \\
\text { mesurée }\end{array}$ & & $\mathrm{H}-\mathrm{Ne}$ \\
\hline & $\begin{array}{l}\text { ingéré } \\
\text { MI } \\
\text { cal. }\end{array}$ & $\begin{array}{c}\text { correspondante } \\
\mathrm{Ms} \\
\mathrm{g}\end{array}$ & $\begin{array}{c}\text { expérimentalement } \\
\mathrm{Ne} \\
\text { cal. }\end{array}$ & $\begin{array}{l}\text { Différence } \\
M-\mathrm{Ne} \\
\text { cal. }\end{array}$ & $\begin{array}{c}\text { Ms } \\
\text { cal. par } g\end{array}$ \\
\hline oine .. & 265 & 88,8 & $\overline{174}$ & $\overline{9 \mathrm{I}}$ & .02 \\
\hline
\end{tabular}

20. - EXPÉRIENCES SUR VOLAIILES EN PONTE E'T A L'ENTRETIEN (A. M. I,EROY et J. Delage. - Annales de la nutrition et de l'Alimentation, 209-244; I954).

Volaille en ponte

\begin{tabular}{|c|c|c|c|c|c|c|c|}
\hline $\begin{array}{c}\text { Poids de } \\
\text { la } \\
\text { volaille }\end{array}$ & $\begin{array}{l}\text { Poids de } \\
\text { l'œuf } \\
\text { l;ondu }\end{array}$ & $\begin{array}{l}\text { Énergie } \\
\text { métabo- } \\
\text { lisable }\end{array}$ & $\begin{array}{l}\text { Mat. sèche } \\
\text { ingérée }\end{array}$ & $\begin{array}{c}\text { Énergie } \\
\text { de } \\
\text { l'entretien }\end{array}$ & $\begin{array}{l}\text { Energie } \\
\text { de la } \\
\text { production }\end{array}$ & $\mathrm{M}-(\mathrm{E}+\mathrm{P})$ & $\frac{M-(E+P)}{M s}$ \\
\hline $\mathrm{kg}$ & $\mathrm{g}$ & cal. & $\mathrm{g}$ & cal. & cal. & $\mathrm{ca}$ & cal. jarg \\
\hline 2,0 & 60 & $\overline{398}$ & II 4 & $\overline{162}$ & $\overline{120}$ & $\overline{116}$ & $\overline{1,02}$ \\
\hline
\end{tabular}

Volaille à l'entretien 


\section{Sixième série}

\section{EXPERIENCES SUR RATS BLANCS}

21. - Expériences de E. B. Forbes, M. Kriss, C. R. Miller.

Institut of animal nutrition. Pensylvania state college. (Journal of Vutrition, 8, 535; I934).

(Données analysées et revues par A. M. Leroy - exprimées en grandes calories).

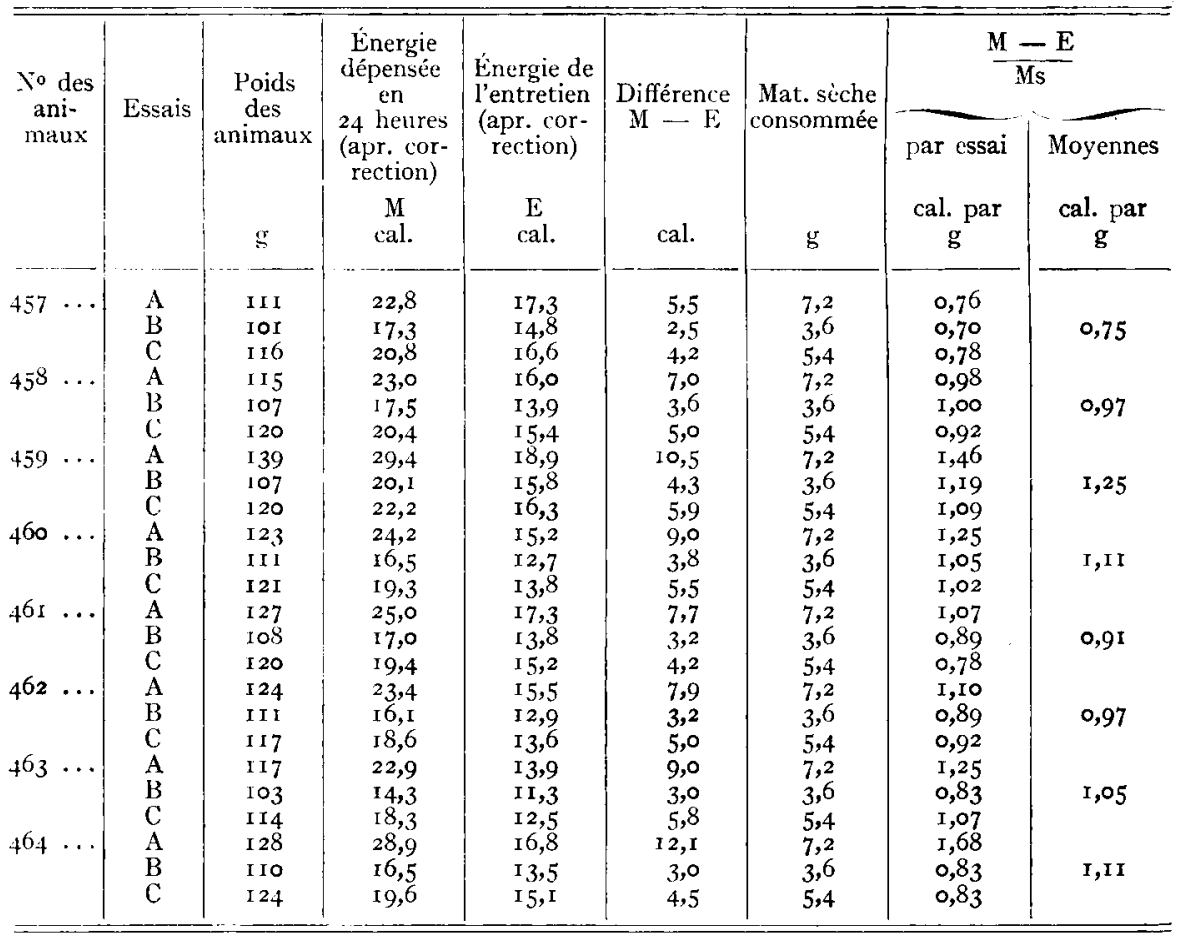

22. - Expériences de E. AARS-JORGEnsen et II. DAM.

Dep. of biocemistry and nutritition - Polytechnie Institut - Copenhague (British Journal of Nutrition, 8, 3; I954).

Résultats moyens pour groupes de six rats chacun

6 DERNIÈRES SEMAINES DE L'EXPÉRIENCE

\begin{tabular}{|c|c|c|c|c|c|c|c|}
\hline $\begin{array}{l}\text { No } \\
\text { de l'essai }\end{array}$ & $\begin{array}{c}\text { Poids } \\
\text { moyen } \\
\text { d'un sujet } \\
\mathrm{g}\end{array}$ & $\begin{array}{l}\text { Mat. sìche } \\
\text { ingérée } \\
\text { Ms } \\
\mathrm{g}\end{array}$ & $\begin{array}{l}\text { Énergie } \\
\text { métaboli- } \\
\text { sable } \\
\text { M } \\
\text { cal. }\end{array}$ & $\begin{array}{c}\text { Énergie } \\
\text { de } \\
\text { l'entretien } \\
\text { F (1) } \\
\text { cal. }\end{array}$ & $\begin{array}{l}\text { Énergie } \\
\text { de la } \\
\text { production } \\
\mathrm{P}\left({ }^{2}\right) \\
\text { cal. }\end{array}$ & $\begin{array}{c}\mathrm{M}-(\mathrm{E}+\mathrm{P}) \\
\mathrm{cal} .\end{array}$ & $\begin{array}{l}\frac{M-(E+P)}{M s} \\
\text { cal. par } g\end{array}$ \\
\hline $\begin{array}{l}\tau \\
2 \ldots \ldots \ldots \\
2 \ldots \ldots \ldots \\
3 \ldots \ldots \ldots \\
4 \ldots \ldots \ldots \\
5 \ldots \ldots \ldots \\
6 \ldots \ldots \ldots \\
7 \ldots \ldots \ldots \\
8 \ldots \ldots \ldots\end{array}$ & $\begin{array}{l}198 \\
213 \\
210 \\
204 \\
226 \\
235 \\
221 \\
238\end{array}$ & $\begin{array}{l}7,3 \\
7,8 \\
6,0 \\
8,6 \\
9,2 \\
7,0 \\
6,5 \\
7,0\end{array}$ & $\begin{array}{l}34,7 \\
37,1 \\
33,6 \\
39,5 \\
39,4 \\
36,8 \\
36,9 \\
37,5\end{array}$ & $\begin{array}{l}25,8 \\
26,8 \\
26,5 \\
25,7 \\
28,2 \\
29,2 \\
27,6 \\
29,6\end{array}$ & $\begin{array}{l}\mathrm{I}, 5 \\
\mathrm{I}, 5 \\
\mathrm{I}, 7 \\
\mathrm{I}, 7 \\
\mathrm{I}, 7 \\
\mathrm{I}, 8 \\
\mathrm{I}, 7 \\
\mathrm{I}, 7\end{array}$ & $\begin{array}{r}7,4 \\
8,8 \\
5,4 \\
12,1 \\
9,5 \\
9,8 \\
5,8 \\
7,6 \\
6,2\end{array}$ & $\begin{array}{l}\text { I,01 } \\
\text { I,I3 } \\
0,90 \\
1,40 \\
1,03 \\
0,83 \\
1,17 \\
0,89\end{array}$ \\
\hline
\end{tabular}

(1) Évalué d'après la table correspondant aux animaux abondamment nourris.

(2) Évalué d'après le gain de poids vif, à raison de 2 cal. par g de gain. 
Comme il est aisé de le constater, les quotients ainsi obtenus se groupent autour d'une même valeur moyenne $\frac{M-(E+P)}{M s}$ qui, pour chacune des espèces animales envisagées, est voisine de I calorie par gramme de matière sèche. Voici, classés par espèces, les résultats de ces calculs, accompagnés de leur moyenne générale.

\section{TABLEAU III}

Valeurs, pour chaque espèce animale considérée, des moyennes du quotient $\frac{\mathrm{M}-(\mathrm{E}+\mathrm{P})}{\mathrm{Ms}}$ exprimé en calories, la matière sèche étant exprimée en grammes.

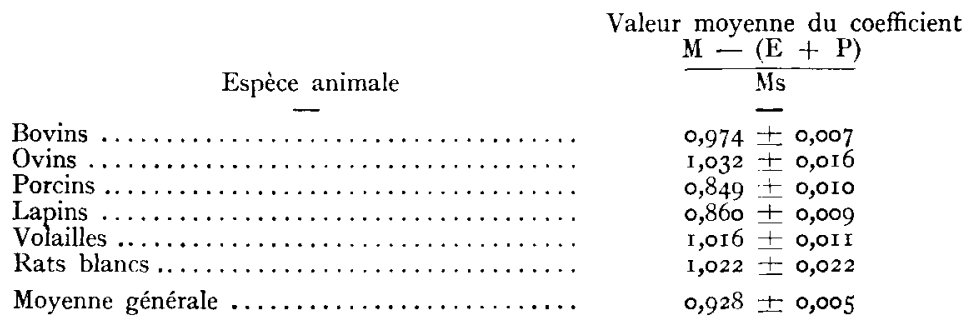

La figure 5 montre comment les données de chacune de ces collections se groupent autour de la valeur moyenne correspondante. La dispersion régulière de ces données semble conforme à la loi de Gauss.

\section{Différences entre les données obtenues avec chaque espèce :}

Si la différence entre les moyennes des résultats observés sur les bovins et les ovins est à la limite de la signification, l'écart que nous pouvons calculer entre la moyenne des porcins et celle des lapins est du même ordre de grandeur que son erreur. Mais l'écart entre le coefficient des bovins et celui des porcins, ainsi que celui qui existe entre les coefficients des bovins et des lapins, sont suffisamment éloignés de leur limite d'erreur pour que nous puissions les prendre en sérieuse considération, comme le montrent les données du tableau 4 :

\section{Tableau IV}

$$
\begin{aligned}
& \text { Ecarts entre les coefficients } \frac{M-(E+P)}{M s} \\
& \text { correspondant aux espèces animales examinées. }
\end{aligned}
$$


Figure 5

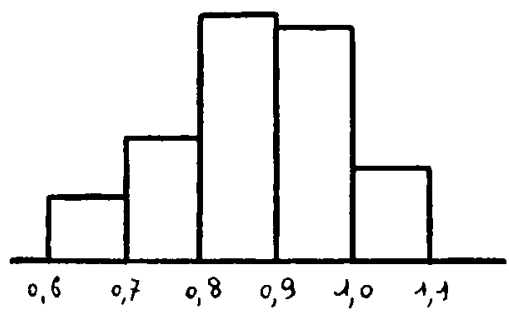

Bovins.

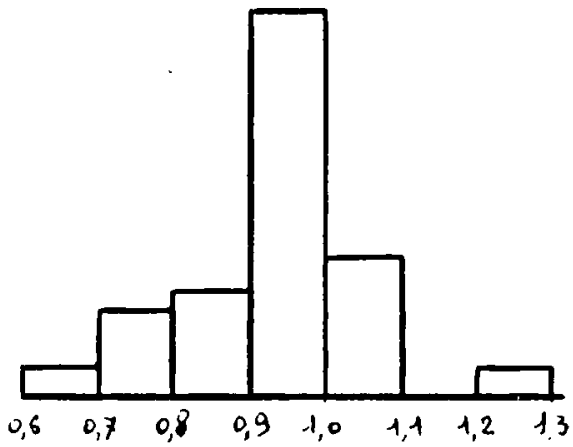

Ovins.
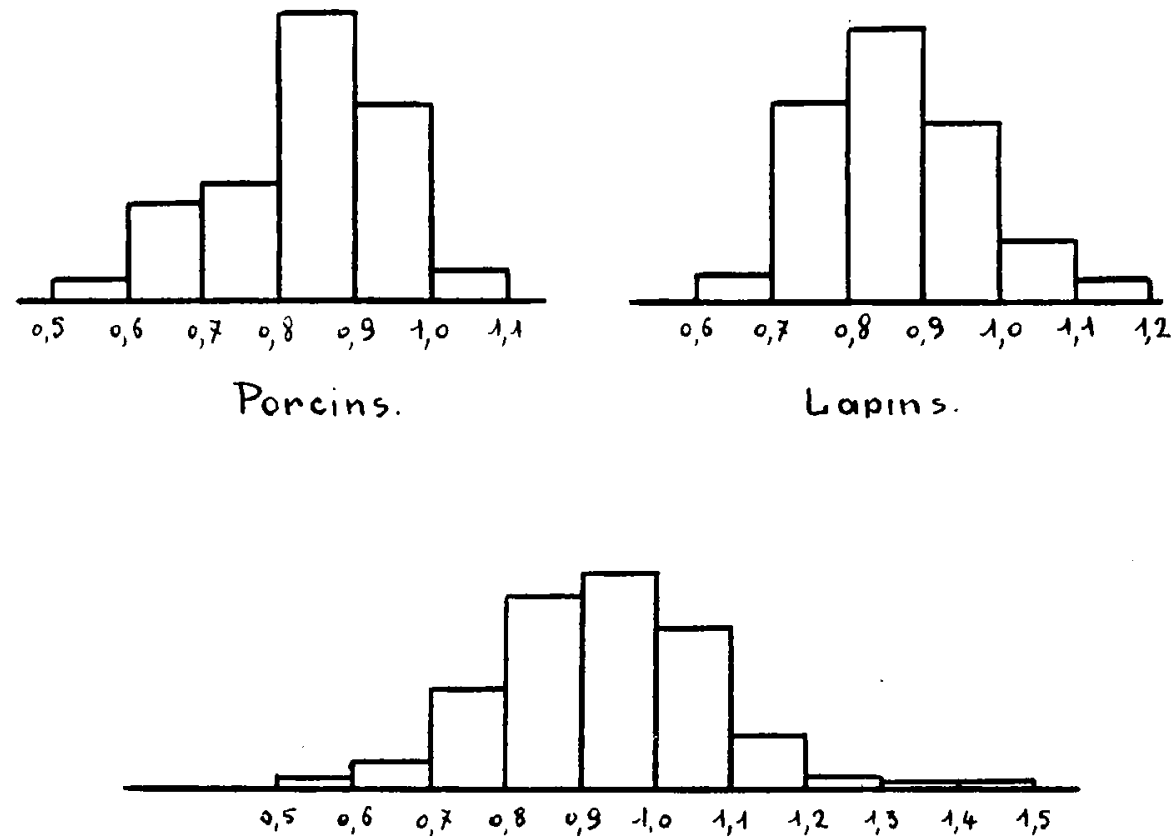

Toutes données réunies. 
Il semble donc exister une différence entre les ruminants et les monogastriques, en ce qui concerne le jeu de l'action dynamique spécifique des aliments, ce qui peut sans doute s'expliquer par la rapidité avec lesquels ces derniers peuvent ingérer leur nourriture. Si les résultats obtenus avec les volailles et les rats paraissent faire exception à cette règle, c'est vraisemblablement parce que le petit nombre des mesures dont nous disposions et aussi les difficultés de celles-ci, ne nous ont pas permis de calculer les indices correspondants avec la même précision que pour les quatre autres espèces. De nouvelles recherches devraient donc être entreprises pour vérifier l'exactitude de cette opinion.

\section{Analyses des résultats obtenus pour chaque espèce :}

Un examen attentif des données que nous avons précédemment reproduites nous montre que le coefficient d'accroissement du métabolisme par rapport à la matière sèche paraît dépendre du rapport de l'énergie nette totale, $\mathrm{E}+\mathrm{P}$, à l'énergie d'entretien $\mathrm{E}$. Pour vérifier le bien-fondé de cette constatation, nous avons calculé le coefficient de corrélation entre les valeurs du rapport $\frac{E+P}{E}$ et celles des rapports $\frac{M-(E+P)}{M s}$ correspondants. Voici, pour les bovins, les porcins et les lapins, les valeurs respectives de ces coefficients de corrélation (tableau 5).

\section{TABleaU V}

Corrélation entre les valeurs des rapports $\frac{M-(E+P)}{M s}$, et les valeurs correspondantes du rapport $\frac{E+P}{E}$

\begin{tabular}{|c|c|}
\hline & Coefficient de corrélation \\
\hline 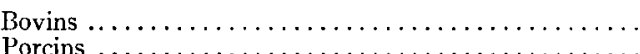 & $+0,221$ \\
\hline $\begin{array}{l}\text { Pran } \\
\text { L }\end{array}$ & $\begin{array}{r}+0,393 \\
\therefore 0,062\end{array}$ \\
\hline
\end{tabular}

Pour les bovins, le calcul de ce coefficient de corrélation pour la seule série des expériences de Forbes donne comme résultat $+0,363$.

S'il existe bien, pour les bovins et les porcins, un coefficient de corrélation nettement positif, la relative faiblesse de ce dernier montre que la règle de proportionnalité qui paraît lier l'augmentation de l'énergie dépensée par gramme de matière sèche et le quotient de l'énergie nette totale à l'énergie de l'entretien souffre de nombreuses exceptions. Cette constatation peut parfaitement s'expliquer si l'on se souvient que ce qui conditionne l'influence de la hausse du métabolisme pendant les repas sur la dépense calorifique totale des 24 heures, c'est essentiellement la 
durée de l'ingestion des aliments. Or, d'un animal à l'autre, la durée d'ingestion d'une même quantité de matière sèche peut parfaitement varier, selon les dimensions de l'armature buccale des animaux et surtout la plus ou moins grande vivacité de leurs mouvements de mastication et de déglutition. Ainsi peuvent s'expliquer les différences qui s'observent sur des animaux de la même espèce, autant que l'on en peut juger par l'examen comparé des données ci-après correspondant à des individus déterminés (tableau VI).

\section{TabienaU VI}

Différence entre les rapports $\frac{M-(E+P)}{M s}$ correspondant à des individus de la même espèce

\begin{tabular}{|c|c|}
\hline & Coefficient moyen $\frac{M-\left(\mathrm{L}+\mathrm{I}^{\prime}\right)}{\mathrm{Ms}}$ \\
\hline Bovins & Calories par $\mathrm{g}$ de matière sèche \\
\hline \multicolumn{2}{|l|}{ Expériences de KeilLner : } \\
\hline Animal $N_{0} 5 \ldots \ldots \ldots \ldots \ldots \ldots \ldots \ldots \ldots \ldots \ldots$ & $\mathrm{I}, 04$ \\
\hline 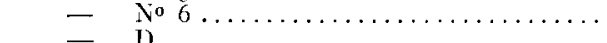 & 1,15 \\
\hline$=\quad D_{1} \cdots \ldots \ldots \ldots \ldots$ & $1, \infty$ \\
\hline 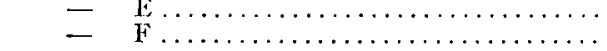 & $\begin{array}{l}\text { I, IO } \\
\mathrm{I}, \mathrm{O}\end{array}$ \\
\hline \multicolumn{2}{|l|}{ Expériences de FingerLing } \\
\hline 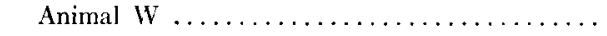 & 1,12 \\
\hline$-17 \ldots \ldots \ldots \ldots$ & $\mathrm{I}, 04$ \\
\hline 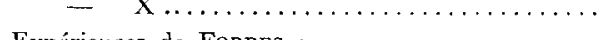 & $\mathrm{I}, \mathrm{06}$ \\
\hline \multicolumn{2}{|l|}{ Expériences de Forbes : } \\
\hline 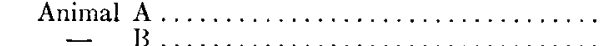 & 0,82 \\
\hline & \\
\hline \multicolumn{2}{|l|}{ Porcins } \\
\hline \multicolumn{2}{|l|}{ Expériences de FingERLING : } \\
\hline Animal $\mathrm{XVI}, \ldots \ldots \ldots \ldots$ & 0,87 \\
\hline$-\quad x V I I \ldots \ldots \ldots \ldots \ldots \ldots \ldots \ldots$ & 0,78 \\
\hline \multirow{2}{*}{\multicolumn{2}{|c|}{$\begin{array}{l}\text { Lapins } \\
\text { Expériences de A. Hellberg : }\end{array}$}} \\
\hline & \\
\hline Animal I $12 \ldots \ldots \ldots \ldots \ldots$ & $1, \mathrm{II}$ \\
\hline $\begin{array}{rr}- & \text { I } 4 . \\
- & \text { I } 74 .\end{array}$ & $\begin{array}{l}0,88 \\
0.99\end{array}$ \\
\hline $\begin{array}{l}-\quad 174 \cdots \\
-\quad 184 \cdots\end{array}$ & $\begin{array}{l}0.99 \\
0,78\end{array}$ \\
\hline
\end{tabular}

\section{Différences obtenues sur des sujets appartenant à la même espèce, observés par des expérimentateurs différents :}

I1 existe des différences significatives entre les résultats obtenus sur des bovins par les auteurs allemands, d'une part, par les auteurs américains et par Mol,GAARD, d'autre part, comme l'indiquent les données du tableau 7 ci-dessous. 


\title{
TABLEAU VII
}

Différences entre les rapports $\frac{M-(E+P)}{M s}$

correspondant aux expériences sur bovins faites par différents auteurs

\author{
Valeurs de $\frac{M-(\mathrm{E}+\mathrm{P})}{M \mathrm{~s}}$ : \\ Calories par $\mathrm{g}$ de m.s. : \\ Expériences de Kei.Lner et de Fingerling : \\ a) $\mathrm{I}, 03 \pm 0,007$; \\ Expériences de Forbes et de Mitchell-Hamilton : b) $0,89 \pm 0,0$ Io; \\ Expériences de MollgaARD : \\ c) $0,93 \pm 0,013$.
}

Différence entre $a$ et $b: 0,14 \pm 0,012$ très significatif ;
Différence entre $a$ et $c: 0,10 \pm 0,015$ très significatif;
Différence entre $b$ et $c: 0,04 \pm 0,016$ non significatif.

Si la différence entre les résultats calculés d'après les données des auteurs allemands et ceux qui concernent les vaches laitières observées par Mol_LGAard peuvent éventuellement s'expliquer par des dépenses énergétiques différentes provenant de la transformation des matériaux nutritifs en matières azotées et grasses, dans le cas de la croissance et de l'engraissement, ou en matières azotées, lactose et matières grasses, dans le cas de la production laitière, on ne saurait expliquer de la même manière la différence constatée entre les données des auteurs allemands et des auteurs américains, qui ont opéré sur des animaux en état d'entretien ou d'engraissement.

Remarquons, tout d'abord, que le rapport moyen $\frac{E+P}{E}$ pour les expériences effecutées par KELLLNER et FiNGERLING s'élève à $I, 55$, alors qu'il n'est que de I,30 pour la série des expériences de Forbes. Mais une observation attentive des conditions expérimentales dans lesquelles ont travaillé ces auteurs peut nous faire comprendre la raison de cette différence. En effet, la durée totale des repas quotidiens d'un animal ne dépend pas seulement de la quantité totale de matière sèche ingérée au cours de ces repas; elle dépend aussi de la structure physique de cette matière sèche, ainsi que de la plus ou moins forte quantité d'eau qui accompagne les matériaux organiques de la ration ingérée.

Tandis qu'il faut à un bovin de 20 à 30 minutes pour absorber un kilogramme de la matière sèche contenue dans le foin, selon la qualité de cet aliment, une quantité analogue de matière sèche provenant d'un aliment concentré présenté sous forme d'agglomérés peut être consommée en moins de dix minutes. Le plus ou moins grand degré d'appétence de l'animal pour les aliments qu'on lui présente exerce également une action sur la durée de consommation de l'ensemble des matériaux nutritifs composant la ration journalière, et cette appétence peut d'ailleurs fort bien ne pas être la même pour des individus d'un même troupeau, 
car tous les animaux ne subissent pas d'une manière uniforme l'effet des excitants, agissant à la fois sur leur vue, leur odorat ou leur goût, qui conditionne le phénomène. Or, les animaux observés par Forbes et ses collaborateurs, d'une part, et par MiTCHELL et HAMILTON, d'autre part, ont reçu des quantités d'aliments concentrés proportionnellement beaucoup plus grandes que celles des bœufs de KELLnER et de Fingeri,ing, ce qui explique fort bien l'existence de la différence entre les indices précédemment constatée.

L'influence exercée par la plus ou moins grande quantité d'eau absorbée avec les aliments peut être mise en évidence, pour les porcins, par la comparaison des données concernant l'alimentation avec sérum de fromagerie, d'une part, et l'alimentation effectuée selon les normes danoises, avec lait écrémé, d'autre part. Pour les porcs nourris au sérum, l'énergie calorifique perdue par gramme de matière sèche ingérée est de 0,96 calories, alors qu'elle n'est que de 0,88 calories pour les porcs nourris d'après la méthode danoise. Tandis qu'un porc de 60 kg nourri au sérum doit absorber I2 à I5 litres de liquide par jour, la ration d'un porc danois limitée à $7-8$ litres de liquide est consommée beaucoup plus rapidement, ce qui réduit la durée pendant laquelle la dépense énergétique de l'animal atteint son niveau maximum, et donne aux porcs ainsi alimentés un net avantage par rapport à ceux recevant du sérum.

Pour la même raison, une ration abondante de pommes de terre cuites est consommée moins vite qu'une quantité équivalente, sous le rapport de l'énergie métabolisable, d'un aliment comportant des cossettes de pommes de terre desséchées. Il en résulte - à quantité égale de matière sèche - une légère réduction de l'équivalent nutritif des tubercules par rapport à la substance obtenue après leur dessiccation préalable. A deux reprises, nous avons apporté nous-mêmes, avec nos collaborateurs R. Feivrier et G. CharieT-I,ERY, des preuves expérimentales de l'influence d'une réduction de la durée de consommation des aliments sur l'efficacité nutritive de ces derniers. Fin opérant avec des porcs, nous avons montré qu'en réduisant la durée totale des deux repas journaliers à 20 minutes, il était possible d'accroitre d'environ $8 \mathrm{p}$. Ioo l'efficacité des matériaux ingérés, par rapport à la même alimentation distribuée ad libitum. Autrement dit, en réglementant strictement la durée de l'accès des animaux à leur mangeoire, sans modifier en quoi que ce soit la quantité journalière de matière sèche ingérée, nous avons constaté qu'il fallait 8 p. roo de matière sèche de moins pour obtenir de nos animaux un accroissement d'un kilogramme de poids vif.

Dans une autre expérience, après avoir constaté que des volailles consommaient plus rapidement une ration composée de grains et de granules qu'une autre composée des mêmes aliments présentés sous la forme de farine grossière, nous avons mesuré exactement, sur deux lots 
de poules aussi identiques que possible, la quantité d'aliments consommée correspondant à la ponte d'un ouf de 60 grammes.

Les résultats obtenus nous ont montré que l'effet nutritif de roo grammes d'une nourriture composée de granulés était entièrement comparable à celui de I Io grammes du même aliment consommés sous forme de mash sec. Tout ceci prouve à l'évidence que la durée de consommation des aliments exerce une action indéniable sur l'utilisation de la quantité d'énergie métabolisable qu'ils apportent. L'éleveur doit donc s'ingénier à rechercher des animaux mangeant le plus vite possible une quantité d'aliments en rapport avec leur capacité de transformation de ces derniers en viande ou en lait. L'expérience montre, par exemple, que les grandes championnes laitières sont douées d'un robuste appétit, que les éleveuts s'efforcent de rassasier au moyen d'aliments ingérés rapidement, sous forme de produits concentrés correctement équilibrés. De même, les porcs gros mangeurs sont ceux qui s'engraissent le plus vite, avec la moindre dépense de matériaux nutritifs par rapport à leur gain de poids vif.

\section{Influence de l'excès de l'alimentation azotée sur la dépense supplémentaire entraînée par la consommation d'un gramme de matière sèche}

Si nous considérons maintenant les résultats des expériences au cours desquelles les animaux ont reçu des quantités de matières azotées digestibles dépassant largement leurs besoins, nous pouvons constater que la dépense supplémentaire d'énergie par gramme de matière sèche, en supplément de l'énergie du strict entretien $\mathrm{F}$, se trouve augmentée dans une notable proportion. Il suffit pour cela d'examiner les données du tableau ci-après :

TABLEAU VIII

\begin{tabular}{|c|c|c|c|c|c|c|c|c|}
\hline Référence & 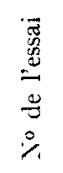 & 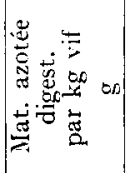 & 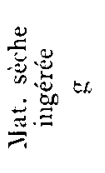 & 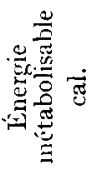 & 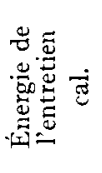 & 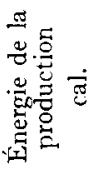 & 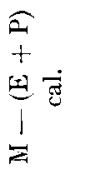 & 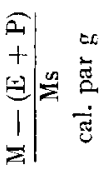 \\
\hline \multicolumn{9}{|c|}{ Expériences de KELLNER et KOHI.ER } \\
\hline \multirow{10}{*}{$\begin{array}{l}\text { Landw. Vers. } \\
\text { Stat. V. } 53\end{array}$} & & 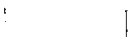 & & & & & & \\
\hline & B I & $\mathrm{I}, 90$ & 12237 & 33455 & 8339 & 8077 & 17039 & I,39 \\
\hline & $\mathrm{B}_{3}$ & 1,77 & 12597 & 34216 & $85^{80}$ & $875^{8}$ & 16978 & $x, 35$ \\
\hline & C 3 & 2,43 & I 2597 & $33 \circ 85$ & 8650 & $974^{8}$ & 14687 & 1,16 \\
\hline & $3-3$ & 1,24 & 8235 & $2237 \mathrm{I}$ & 8710 & 4077 & I 2787 & 1,16 \\
\hline & $3-4$ & $\mathrm{~J}, 96$ & $893^{\circ}$ & 25068 & 8920 & 5287 & $1086 \mathrm{I}$ & $\mathrm{I}, 2 \mathrm{I}$ \\
\hline & $4-3$ & $\mathrm{I}, 35$ & 8410 & 22697 & 8310 & 4010 & 10377 & 1,23 \\
\hline & $\mathrm{D}-t$ & 2,34 & 12371 & 32229 & 9880 & 8221 & I 4 I 28 & $\mathrm{I}, \mathrm{I} 4$ \\
\hline & $\mathrm{B}-4$ & 2, I 2 & 10618 & 26703 & 9600 & 3 I $4^{6}$ & I3957 & I, 3 I \\
\hline & $J-2$ & I,61 & 10205 & $25^{8} 3^{6}$ & 8270 & 5205 & $123^{6 \mathrm{I}}$ & 1,20 \\
\hline \multicolumn{9}{|c|}{ Expériences de FiNGERLING } \\
\hline \multirow{3}{*}{$\begin{array}{l}\text { Landw. Vers. } \\
\text { Stat. I } 8 \text {-I } 19\end{array}$} & & & & & & & & \\
\hline & 16,3 & 2,35 & 9459 & 25360 & 9300 & 3321 & I 2739 & 1,34 \\
\hline & 16,4 & 2,57 & $945^{\circ}$ & 24659 & 9310 & $323^{8}$ & I 2 I I & 1,28 \\
\hline
\end{tabular}


Avec l'ensemble des données de KEL,LNER et KOHLER, il est possible de montrer l'existence d'une corrélation positive entre l'excédent de dépense calorifique par gramme de matière sèche ingérée et la teneur de la ration en matière azotée totale digestible par kilogramme de poids vif des animaux. Le cofficient de corrélation correspond est de $+0,498$.

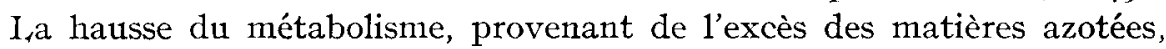
est nettement supérieure à celle que 1'on pourrait obtenir ell appliquant la loi formulée par Borsoock, d'après laquelle cet accroissement de perte énergétique serait, comme nous l'avons rappelé précédemment, de 8,4 c par gramme d'azote excédentaire éliminé par l'urine. Il semble donc bien que les matières azotées distribuées en excédent stimulent certaines fonctions organiques, et diminuent de ce fait fortement le coefficient d'utilisation de l'énergie métabolisable. Il convient donc, pour obtenir le maximum d'efficacité des aliments distribués, de respecter scrupuleusement les règles de l'alimentation rationnelle, qui sont, rappelons-le, les suivantes :

\section{'TABLEAT IX}

Règles à respecter pour éviter à la fois l'insuffisance et l'excès des matériaux azotés de la ration alimentaire

\footnotetext{
Quantité de matière azotée exprimée, pour les ruminants, en équivalents protéiques, et, pour les monogastriques, en matières protéiques digestibles :

Animaux à l'entretien : $0,5 \mathrm{~g}$ par $\mathrm{kg}$ de poids vif ;

Animaux adultes, à l'engraissement : $1,0 \mathrm{~g}$ par $\mathrm{kg}$ de poids vif.

l'our la production laitière, en supplément de la ration d'entretien, par $\mathrm{kg}$ de lait produit

Vaches laitières : $60 \mathrm{~g}$;

Brebis et truies : 100 à $\mathbf{1} 20 \mathrm{~g}$.
}

\section{Utilisation pratique des données précédentes :}

De l'importante documentation qui précède, il est possible de déduire une méthode d'alimentation, qui possède l'avantage d'être à la fois moins empirique et plus facile à exposer que les méthodes faisant appel à la conception de l'énergie nette, dont le prototype est celle des équivalents en amidon de KELINER.

En effet, l'équation fondamentale de l'équilibre énergétique d'un animal, placé dans des conditions de température telles que ni la chaleur ni le froid excessifs ne peuvent intervenir sur son métabolisme, s'énonce, nous l'avons vu, de la manière suivante :

\begin{tabular}{|c|c|c|c|}
\hline & $=.$. & $\mathrm{K}$ Ms & + \\
\hline étal & de l'eir & (m). & $\begin{array}{r}\text { (Ene } \\
\text { des prod }\end{array}$ \\
\hline
\end{tabular}

dans laquelle la valeur de $\mathrm{K}$ est égale, pour simplifier, à $\mathrm{r}, \mathrm{o}$ pour les ruminants et les volailles, et à 0,9 pour les lapins et les porcs. 
A partir des teneurs en matière organique digestible des aliments qui figurent dans les tables usuelles, nous pouvons calculer rapidement la quantité d'énergie métabolisable correspondante. Il suffit pour cela d'ajouter au total de la matière organique digestible contenue dans un kilogramme de l'aliment considéré, le résultat de la multiplication de la quantité de matière grasse digestible par le cœefficient $\mathrm{I}, 25$. On obtient ainsi la somme des " éléments digestibles totaux " désignés par les auteurs américains sous le nom de T. D. N. (Total digestible nutrients). La somme en question, multipliée par 3,65 (cas des ruminants), 4, I (cas des porcs, des jeunes ruminants non sevrés et des volailles), ou par 4,2 (cas des lapins), fournit une mesure suffisamment précise de l'énergie métabolisable, comme il est possible de s'en assurer par la comparaison des mesures directes de cette énergie effectuées par KELL,NER, FingERLiNG, MollgaArd, LEROY-ZELTER, HeLlbERG, aux quantités de matières organiques digestibles correspondantes :

\section{TABLEAU $X$}

Justification des cofficients à utiliser pour le calcul de l'éneraie métabolisable à partir des éléments digestibles totaux

\begin{tabular}{|c|c|c|c|}
\hline Expérimentateurs & Espèce animale envisagée & $\begin{array}{c}\text { Nombre } \\
\text { d'expériences } \\
-\end{array}$ & $\begin{array}{l}\text { Coefficient de } \\
\text { multiplication } \\
\end{array}$ \\
\hline KHLLNER et KOHLER.... & Bovins . & 39 & $3,59 \pm 0,009$ \\
\hline Fingerling . . . . . . . . & Bovins & 28 & $3,68+0,010$ \\
\hline MOLLGAARD) & Vaches laitières $\ldots \ldots \ldots \ldots \ldots \ldots$. & 15 & $3,69 \pm 0,010$ \\
\hline & Moyenne pour les expériences sur bovins. & 92 & $3,64 \pm 0,006$ \\
\hline LIROY Z-ZELTE & Agneaux rêcemment sevrés ........ & 3 & $4,10 \pm 0,056$ \\
\hline LeROX-ZELTER . . . . . . . & 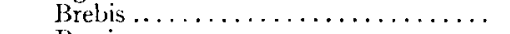 & 4 & $3,69 \pm 0,029$ \\
\hline Fingerling $\ldots \ldots \ldots \ldots$ & Porcins $\ldots \ldots \ldots \ldots \ldots \ldots \ldots \ldots$ & $3^{2}$ & $4,12 \pm 0,003$ \\
\hline HHLLBLRG $\ldots \ldots \ldots \ldots$ & $\ldots \ldots \ldots \ldots \ldots \ldots$ & 67 & $4,19 \pm 0,008$ \\
\hline
\end{tabular}

D'autre part, lorsque l'on connait le poids d'un animal, il est aisé de déterminer l'importance de ses besoins d'entretien, exprimés en grandes calories, à l'aide du tableau 2. Il suffit ensuite de savoir quel est, exprimée en calories, la quantité de produit que l'on veut obtenir, pour calculer la masse des aliments nécessaires à l'animal. Voici, au moyen d'un exemple simple, comment on peut de cette manière établir un rationnement.

Supposons que nous voulons connaitre le poids de foin de pré de très bonne qualité nécessaire au maintien en équilibre énergétique d'une vache laitière de $600 \mathrm{~kg}$ donnant $\mathrm{I} 5 \mathrm{~kg}$ de lait à $4 \mathrm{p}$. Ioo de matière grasse, dont la valeur calorifique est de $75^{\circ}$ calories par $\mathrm{kg}$. Le foin que nous cherchons à utiliser contient $535 \mathrm{~g}$ de matière organique digestible par $\mathrm{kg}$ et sa teneur en matière grasse digestible est de $15 \mathrm{~g}$ par $\mathrm{kg}$. Un kilogramme de ce foin fournit à l'animal une quantité d'énergie métabolisable de $(535+\mathrm{I} 5 \times 1,25) \times 3,65=2$ o20 calories, pour $840 \mathrm{~g}$ de matière sèche. 
L'équation qui nous donne la solution du problème présente la forme suivante, dans laquelle $x$ figure la quantité de foin cherchée, exprimée en kilogrammes :

d'où

$$
\underset{\mathbf{M}}{2020} x \underset{\mathrm{E}}{8000}+\underset{\operatorname{Is}}{840 x}+\underset{\mathrm{P}}{\operatorname{II}} 250
$$

$$
x=\mathrm{I} 6,3 \mathrm{~kg} \text { de foin. }
$$

Des problèmes plus compliqués peuvent être résolus de cette manière. Ainsi, par exemple, il suffit de connaître la composition de la ration de base composée de produits de la ferme, distribuée à des vaches, pour être en mesure de calculer la quantité d'énergie métabolisable que représente cet apport. Avec des aliments concentrés appropriés, il est facile de composer une formule de mélange, équilibrée en matières azotées digestibles et en matières minérales, et de déterminer la quantité d'énergie métabolisable correspondant à un kilogramme de cette dernière. Un calcul analogue au précédent permet dans ces conditions d'obtenir rapidement la quantité de ce mélange à utiliser pour satisfaire les besoins d'énergie d'une vache dont on connaît le poids et la production quotidienne.

\section{Le problème des substitutions alimentaires :}

Imaginons qu'un animal, en parfait équilibre énergétique, reçoive une ration de base $\mathrm{R}$, complétée par un kilogramme d'un aliment de référence A. L'équation qui rend compte de cet équilibre s'écrit de la manière ci-après en choisissant le coefficient de multiplication de la matière sèche égal à I (cas des ruminants).

$$
\mathrm{R}+m=\mathrm{E}+\mathrm{Ms} \times 0,9+m s \times 0,9+\mathrm{P}
$$

dans laquelle :

$R$ représente la quantité d'énergie métabolisable de la ration de base:

$\mathrm{m}$ représente la quantité d'énergie métabolisable apportée par I $\mathrm{kg}$ de $\mathrm{A}$;

Ms représente la quantité de matière sèche de la ration de base :

ms représente la quantité de matière sèche apportée par I $\mathrm{kg}$ de $\mathrm{A}$;

$\mathrm{E}$ représente le besoin d'entretien de l'animal;

$P$ représente le besoin de production;

I,o est le coefficient de multiplication de la matière sèche.

Nous voulons calculer la quantité $x$ d'un aliment B, qui, substituée à un $\mathrm{kg}$ de $\mathrm{A}$, continue à maintenir l'animal dans les mêmes conditions, c'est-à-dire qui permet de satisfaire les mêmes besoins d'énergie nette, $\mathrm{E}+\mathrm{P}$.

Appelons m' l'apport d'énergie métabolisable par $\mathrm{kg}$ de $\mathrm{B}$, et $m s^{\prime}$ la quantité correspondante de matière sèche.

La nouvelle équation d'équilibre devient après la substitution :

$$
\mathrm{R}+m^{\prime} x=\mathrm{E}+\mathrm{Ms} \times \mathbf{I}, 0+m s^{\prime} \times 0,95 \times x+\mathrm{P} .
$$


Retranchons membre à membre l'équation 2 de la première; il vient :

$$
m-m^{\prime} x \cdots\left(m s-m s^{\prime} x\right) \times 1,0
$$

de laquelle nous tirons :

$$
x=\frac{m^{\prime}-m s^{\prime}}{m-m s} .
$$

A titre d'exemple, calculons par cette méthode la quantité de foin de pré de bonne qualité (celui de l'exemple précédent), qui pourrait être substituée à un kilogramme d'orge moyenne, dans la ration d'un ruminant.

Les éléments de ces calculs sont les suivants :

\section{'TABLFAU XI}

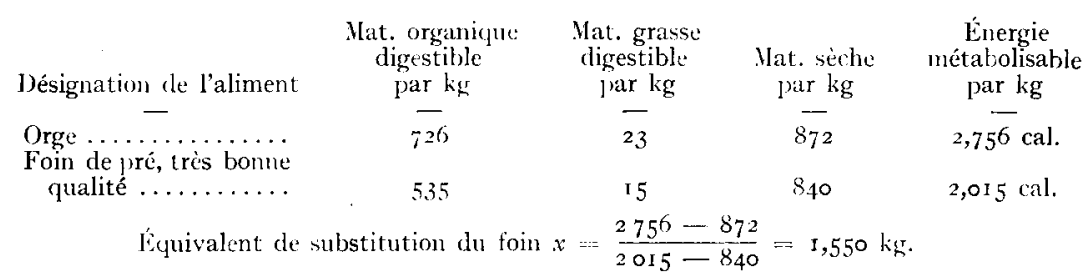

Mais 1'équilibre ainsi établi n'existe qu'à la condition expresse que l'alimentation azotée de notre animal demeure également en parfait équilibre. S'il n'en était pas ainsi, l'excès de la matière azotée distribuée, par le jeu de l'action dynamique spécifique provenant de l'azote excédentaire, occasionnerait une perte supplémentaire qui ferait disparaître l'équilibre de la balance énergétique. I)ans le cas de la substitution du foin de pré à l'orge, nous augmenterions ainsi la quantité de matières azotées digestibles de 84 grammes, correspondant à I3, 3 g d'azote. I.'élimination de cet azote par l'urine entrainerait théoriquement une perte d'énergie de II 2 calories $(\mathrm{I} 3,3 \times 8,4)$. Après correction, pour tenir compte de cette contingence, l'équivalent du foin se trouverait ainsi ramené à $\mathrm{I}, 800 \mathrm{~kg}$.

Pratiquement, à la condition d'utiliser deux ou plusieurs substitutions combinées de telle manière qu'elles n'apportent aucun changement à la quantité totale de matières azotées digestibles disponible pour les animaux, convenablement équilibrée auparavant, les divers aliments peuvent se substituer proportionnellement aux quantités calculées par la méthode précédente. Il est aisé de constater que les équivalents déterminés de cette manière correspondent très exactement aux données essentielles du système des unités fourragères scandinaves, dont la simplicité de conception et les modalités d'emploi se prêtent fort bien à la vulgarisation et qui demeurent toujours pour cette raison hautement recommandables. 


\section{Application pratique éventuelle des considérations qui précèdent}

L'action dynamique spécifique des aliments ingérés, proportionnelle à l'ingestion de la matière sèche, est la source d'une production de chaleur que l'animal peut utiliser, lorsqu'il s'agit pour lui de lutter contre le froid. Dans le cas de la stabulation libre, par exemple, il est logique de conseiller d'accroître les quantités des aliments grossiers distribuées et, dans la mesure du possible, de multiplier le nombre des repas, puisque chaque fois que 1'animal mange, l'accroissement de ses activités métaboliques le réchauffe en augmentant son bien-être. Il serait même très utile de placer l'heure du premier repas au moment où la température est la plus froide, c'est-à-dire avant la naissance du jour. Les hygiénistes qui se sont intéressés au problème de l'alimentation de l'homme dans les régions glaciaires préconisent aujourd'hui de fractionner en cinq ou six repas quotidiens d'importance égale les aliments à consommer dans ces conditions, ce qui peut être considéré comme une élégante preuve de la valeur pratique des observations précédentes.

Inversement, dans les pays tropicaux, il est utile de donner aux vaches laitières des repas limités à deux au maximum, composés pour les $3 / 4$ d'aliments concentrés, car la modification obtenue dans ces conditions de la matière sèche ingérée par rapport à l'énergie métabolisable nécessaire réduit de plusieurs milliers de calories la quantité de chaleurdéchet dont l'organisme animal doit se débarrasser, par un mécanisme énergétiquement dispendieux, lorsque la température du milieu qui l'entoure dépasse $25^{\circ}$. Il est recommandable également d'aíimenter les animaux de préférence pendant. la nuit, au moment où la température extérieure est à son plus bas niveau. Des observations exactement conformes à cette manière de voir ont été faites par un zootechnicien hollandais à l'occasion des Journées d'études organisées en septembre I954 à I,ucerne par la Fédération Européenne de Zootechnie.

\section{CONCLUSION}

La méthode de calcul des rations faisant l'objet de ce mémoire possède, sur les procédés utilisés jusqu'alors, un certain nombre d'avantages. Tout d'abord, elle s'applique de la même manière à toutes les espèces animales, quel que soit le format des individus qu'il s'agit de nourrir, ce qui lui confère une très remarquable unité. Ellle se rapproche, en outre, par sa conception, des expériences de mesures directes du métabolisme, car le total de l'énergie d'entretien augmenté de la dépense supplémentaire provenant de la matière sèche ingérée représente la perte d'énergie de l'animal, telle qu'elle pourrait être mesurée par calorimétrie 
directe ou indirecte. lille présente aussi l'avantage de nous faire comprendre les raisons pour lesquelles des animaux de la même espèce et de la même race, soumis à la même alimentation, peuvent tirer de celleci des effets différents. Si nous possédions le moyen de mesurer par un procédé simple et relativement précis le cœefficient de dépense supplémentaire correspondant à l'ingestion d'un gramme de matière sèche, nous aurions le moyen de sélectionner nos animaux d'après ce précieux indice, et de trouver des groupes d'individus caractérisés par la possibilité de s'accroître ou de produire du lait avec une dépense d'aliments inférieure à celle de la moyeme des autres, ce qui pourrait devenir la source d'économies sensibles. L'étude attentive du phénomène de hausse du métabolisme pendant la durée des repas pourrait nous permettre d'en analyser les causes, et d'augmenter nos connaissances encore fragmentaires du mécanisme complexe de l'action dynamique spécifique des aliments. Elle ouvrirait ainsi un champ de recherches d'un intérêt scientifique évident, et pourrait aboutir très rapidement à des applications pratiques capables de rendre de grands services aux éleveurs.

\section{RESUME}

Io L'étude de 337 expériences de métabolisme énergétique, entreprises sur les animaux de six espèces différentes, par I2 groupes d'auteurs allemands, américains, anglais, français, et scandinaves, montre que la chaleur perdue en 24 heures par un animal normalement alimenté, placé dans des conditions d'équilibre, est égale à la dépense d'entretien augmentée d'autant de fois $k$ calories que la nourriture absorbée renferme de grammes de matière sèche.

$2^{0}$ I a valeur de ce cofficient $k$, qui mesure l'action dynamique spécifique des aliments, considérée dans son ensemble, est, pour toutes les espèces examinées, voisine de I calorie par gramme de matière sèche.

Les mesures dont nous donnons le détail dans notre mémoire, ont fourni les résultats ci-après :

Accroissement de l'action dynamique
Jispécificue provenant des aliments ingérés,
par g de matière sèche.

$3^{0}$ Pour les ruminants et les porcs, il existe une tendance à 1'accroissement de ce cœfficient lorsque le rapport de l'énergie nette totale $(\mathrm{E}+\mathrm{P})$ à l'énergie d'entretien $\mathrm{E}$ tend lui-même à augmenter. Ite cœeff- 
cient de corrélation entre la grandeur de l'indice $k$ et le rapport $\frac{(\mathrm{E}+\mathrm{P})}{\mathrm{E}}$ est de $+0,22$, pour les bovins, et de $+0,39$, pour les porcins.

$4^{\circ}$ L'explication de cette dernière constatation pourrait en partie provenir de l'existence d'un accroissement de la dépense d'entretien lorsque la valeur énergétique nette totale de la ration $(\mathrm{E}+\mathrm{P})$ tend à s'éloigner notablement de E;.

$5^{\circ}$ Il existe entre les coefficients $k$ mesurés sur certains individus appartenant à une même espèce des différences notables, ce qui donne à penser que l'on peut trouver, parmi les animaux d'une même troupeau, de bons et de mauvais utilisateurs d'aliments.

$6^{\circ}$ La grandeur du coefficient $k$ est en relation avec la durée totale des repas journaliers de 1'animal. Elle a tendance à diminuer quand l'animal consomme sa ration avec une grande rapidité, pendant des périodes de temps limitées volontairement par l'éleveur.

$7^{\circ}$ A l'aide de la détermination de l'énergie métabolisable correspondant à une ration donnée, et par la connaissance de la quantité totale de matière sèche contenue dans les aliments ingérés, on peut aisément vérifier qu'un animal dont le besoin d'énergie nette totale $\mathbf{E}+\mathbf{P}$ est connu se trouve ou non en état d'équilibre énergétique. - I e calcul des rations effectué d'après cette méthode donne dans la pratique d'excellents résultats.

$8^{\circ}$ Il serait utile d'entreprendre des expériences systématiques avec les aliments des différentes catégories (foins, ensilages, racines, tubercules, aliments concentrés) distribués isolément ou en mélange, afin de déterminer 1'influence des structures physiques et chimiques de ces fourrages sur leur vitesse de consommation calculée par gramme de matière sèche. Ces essais devraient être effectués au début, au milieu, et à la fin des repas, de manière à pouvoir rechercher dans quelle mesure l'état de replétion de l'estomac conditionne la vitesse de l'absorption de cette matière sèche.

(Reçu en publication le 23 nov. 1954).

\section{BIBLIOGRAPHIE}

Axeison (J.). - Wiss. Abhandung des deut. Akademic der Land. Wissenschaften zu Berlin, BV, 2, I954.

Axeisson (J.), Ariksson (S.). - Annals of the Royal A gric. College of Sweden, vol. 20.

BaChMANn (F.). - C. R. VIII e Congrès Intern. d'Aviculture.

BARBORIAK (J.). - These; Imp. Brunner et Bodmer, Zürich, I953.

BLAXTER (K. L.). - Nutr. Abstr. and Review, 20, 50, I, I949.

BLAXTER (K. L.). - Journal of Nutrition, 6, I2, I952.

BORSOOK-KEIGHLY. - Science, I7, 75, I933.

Borsook-Winegarden. - Proc. Nat. Ac.Sc. i6, 559, i93o; Proc. Nat. Ac. Sc. I7, 3, I93I ; Proc. Nat. Ac. Sc. I7, 75, I931. 
BREIREM (K.). - 162 Beretn. fra Forsogslaborat. Kobenhavn. Tierernährung, 11, 487, 528. Tids fordet Norske Landbruks, 60, 25, 52, 1953.

Brody (S.). - Un. of Missouri. Agr. Exp. Station. Res. Bulletin, 96, 143, 176, I80, I90, I93, 220, 223, 238, I926 à I934.

FINGERling (G.). - Land. Versuchs Stat., II3, I, I932; Land. Versuchs Stat., II4, I, I933; Land. Versuchs Stat., II6, I, I933 ; Land. Versuchs Stat., II8-II9, I, 307, I933; Land. Versuchs Stat., I20-I2I, I, I934.

FISSMER (F. E.). - Zeitsch. für Tierernährung und Futtermittelkunde, 5, I, I09, I94I.

Fraps (G. S.). - Texas A gr. Exp. Stat. Bull. 625, 1942.

Forbes (E. B.), Braman (W. W.), Kriss (M.). - Journal of Agr. Res. 35, 233; I928; Journal of Agr. Res. 40, 37, I930; Journal of Agr. Res. 43. II, I05; I93 I Journal of Agr. Res.46, 8, 753 ; I933.

Forbes (E. B.), KrIS (M.), MILLER (R. C.). - Journal of Nutr., 8, 5-535 ; I934.

GOLDSTEIN (S.). - These. Inst. für haustierernährung an der E.T.H., Juris Verlag, Zürich ; I950.

Hansen-Larsen (L.), Clausen (H.), Jespersen (J.). - C. R. VI e Congrès Intern. de Zootechnie, Copenhague ; I952.

Hellberg (A.). - - Thèse. Lib. Almquist et Wiksells, Uppsala ; I949.

JORGENSEN (E. A.), DAM (H.). - British Journal of Nutr., 8, 3, I954.

Keliner (O.) KoHLER. - Land. Vers. Stat., 53-I-I900.

Kletber (M.). - Schweiz. land. Monashefte, 8; 13, I935; $33^{\mathrm{e}}$ Ann. Proc. of the amer. Soc. of Animal Production; 1940.

LEHMANN (F.). - Zeitschrift für Tierernährung und Futtermittel kunde, I55I73; I94I.

IEROY (A. M.). - C. R. Ve Congrès Intern. de Zootechnie, Paris ; 1949.

LEROY (A. M.). - Ann. de Zootechnie, Sup. I, 47 ; 1952.

L,eroy (A. M.), Charl.eT-Lery (G.), ZeiTER (Z.). Ann. de Zootechnie, 3 , I69; I954.

LAROY (A. M.), Deidage (J.). - Ann. de la Nutrition et de l'Alimentation, $209 ; 1954$.

I ERoy (A. M.), FÉvrier (R.). - Annales Agronomiques, 5 ; I947; Annales agronomiques, 4 ; I949.

LEROY (A. M.), LERY (G.). - Annales Agronomiques, I; 1946; Annales de Zootechnie, I ; 1952 .

LEROY (A. M.), Zeiter (Z.). - Publ. Conseil Supérieur de l'Elevage, Paris: 1936; Annales de Zootechnie, I, 6I-77; 1952. Annales de Zootechnie; I955 (à paraître).

Meigs, Grant. - Proc. Amer. Soc. of Animal Prod., I8; 1936.

Mitcheli (H. H.), Hamilton (T. S.). - Journal of A gr. Res. ,45, 3 ; 1932.

MoligaARD (H.). - Futterungslehre des Milchviehs, Lib. Schäper, Hanovre; 1929. Wissench. Abhaudung des deuts. Akademie der Landwirts. wissenschaften zu Berlin, 5, 2 ; I954.

RuBNER. - Handbuch der Normalen und pathol. Physiologie, I39; 1928.

Schurch (A.). - Schweiz. Landw. Monatshefte, I8, 2 ; 1949.

Vorsin (A.). - Ann. de Zootechnie, sup. I, I ; I952. 